\title{
I. Memoria - Identität - Erinnerungsräume: Methodische und inhaltliche Vorbemerkungen
}

Zwischen der Mitte des 3. und dem ausgehenden 5. Jh. n. Chr. veränderte sich das Erscheinungsbild Roms grundlegend. Trotz der konservierenden Bemühungen der römischen Aristokratie waren um 500 die profanen Bauwerke der Stadt zunehmend von Auflassung und Zerfall bedroht. Es dauerte zwar noch 100 Jahre, bis erstmals ein römischer Tempel in eine Kirche umgewandelt wurde, doch die Zentren des städtischen Raums und der städtischen Kommunikation hatten sich bereits erkennbar verschoben. Die Basiliken der drei großen Heiligen der Stadt, Petrus, Paulus und Laurentius, bildeten seit dem ausgehenden 5. Jh. liturgische und siedlungsgeographische Pole außerhalb der städtischen Mauern, die mit der Stadt durch Portiken verbunden blieben. Parallel zu dieser Verschiebung des Stadtbildes entvölkerte sich der Bereich intra muros zunehmend: Die abnehmende Bevölkerung konzentrierte sich auf bestimmte Viertel, der innerhalb der Stadt gelegene Raum verlor an Kohärenz. Die Prozessionen, mit denen der römische Bischof die Stadt durchzog, um an besonderen Festzeiten den bischöflichen Gottesdienst außerhalb der Lateranbasilika abzuhalten, waren, anders als etwa die Prozessionen in der Nea Roma am Bosporus, nicht darauf ausgerichtet, den städtischen Raum zu erfassen: Der Bischof durchquerte Rom, einen Zwischenraum, auf dem Weg zu den zahlreichen liturgischen Zentren und Inseln der städtischen Kirchen.

Mit diesen räumlichen Veränderungen verbanden sich auch Transformationen städtischer Identität: Die Stadt Rom war um 500 nicht nur in ihrem äußeren Erscheinungsbild nicht mehr dieselbe wie noch rund 250 Jahre zuvor. Ausgehend vom Heiligenkult, der das räumliche Erscheinungsbild des spätantiken Rom zunehmend bestimmte, versucht die vorliegende Studie, anhand der Erinnerung an die Heiligen identitätsrelevante Wandlungsprozesse im städtischen Kontext deutlich zu machen. Ihr Gegenstand ist die Frage, inwieweit die stadtrömische Heiligenerinnerung zwischen dem 3. und 5. Jh. als monumentaler und räumlicher Kristallisationspunkt für die Konstruktion kollektiver Identitäten fungierte, und welche Veränderungen sich in diesem Zeitraum vollzogen.

Die damit berührten Kategorien „Erinnerung“ und „kollektive Identität“, ihre Relation zueinander sowie ihre Bezogenheit auf „Monumente“ und 
„Räume“ verlangen zunächst nach begrifflichen und konzeptionellen Klärungen. Nach diesen methodischen Vorbemerkungen und einer Verortung des hier verfolgten Ansatzes im Kontext der kulturwissenschaftlichen Erinnerungsforschung, werden die inhaltlichen Untersuchungsbereiche näher erläutert, die sich aus seiner konkreten Anwendung auf das Thema des Heiligenkults im spätantiken Rom ergeben.

\section{1. Methodische Vorbemerkungen}

Erinnerung und kollektive Identität in ihren unterschiedlichen Ausprägungen und Repräsentationen (Ethnizität, Kultur, Religion) sind wissenschaftliche Konjunkturbegriffe, ${ }^{1}$ die in den letzten Jahren unübersehbare Spuren in der Landschaft kulturwissenschaftlich ausgerichteter Publikationen hinterlassen haben. Dies gilt nicht zuletzt auch für den althistorischen Bereich, wo neben einer wachsenden Zahl von Einzelstudien ${ }^{2}$ sich insbesondere die Zweite Sophistik $^{3}$ und die Geschichtskultur des republikanischen Rom ${ }^{4}$ zu Forschungsfeldern entwickelt haben, in denen die identitätsstiftende Bedeutung der Erinnerung für Kollektive systematisch untersucht worden ist. Auch in konzeptioneller und methodischer Hinsicht sind zunehmend Beiträge der klassischen Altertumswissenschaften zu verzeichnen. ${ }^{5}$ Dieser regelrechte „Boom“ einer Erinnerungs- und Identitätsforschung verbindet sich zugleich mit einer grundlegenden Neuorganisation der Wissenschaftslandschaft, die sich seit einigen Jahren unter dem Stichwort einer kulturwissenschaftlichen Wende vollzieht und den Anspruch erhebt, unter den Vorzeichen transdisziplinärer Begriffsbildung Offenheit für eine Methodenpluralität zu schaf-

$1 \quad$ Zum Befund und dem Versuch einer wissenschaftsgeschichtlichen Einordnung vgl. zuletzt Cornelißen 2003, 548-555; zu den Gründen für die Konjunktur der Erinnerungsforschung seit den letzten zwanzig Jahren vgl. ferner W. Weber 2003, bes. 16-20.

2 Vgl. u. a. Constructing Identities in Late Antiquity, hrsg. von Richard Miles, London 1999; Ethnicity and Culture in Late Antiquity, hrsg. von Stephen Mitchell/Geoffrey Greatrex, London 2000; Hans-Joachim Gehrke, Mythos, Geschichte und kollektive Identität. Antike exempla und ihr Nachleben, in: Mythen, Symbole und Rituale. Die Geschichtsmächtigkeit der Zeichen in Südosteuropa im 19. und 20. Jahrhundert, hrsg. von D. Dahlmann/W. Potthoff, Frankfurt a. M. u. a. 2000, 1-24; Eckhard Stephan, Honoratioren, Griechen, Polisbürger. Kollektive Identitäten innerhalb der Oberschicht des kaiserzeitlichen Kleinasien, Göttingen 2002.

3 Zu nennen sind hier insbesondere die Arbeiten von Susan Alcock, die die Vergangenheitsbezüge der Zweiten Sophistik auf das Klassische Griechenland anhand der Monumente und Landschaften untersucht und für Fragen der kollektiven Identitätsstiftung fruchtbar zu machen versucht (vgl. Alcock 2001; dies. 2002, 36-98).

$4 \quad$ Flaig 1995; Hölkeskamp 1996 und 2001; T. Hölscher 2001; Walter 2001 und 2004.

5 Zu Identität vgl. zuletzt Gehrke 2004, 361-374. Eine aufschlussreiche methodische Auseinandersetzung mit der Begrifflichkeit „Kultur“ bzw. „Akkulturation“ und den damit verbundenen Fragen der Konstruktion von Identität bietet Gotter 2001. 
fen sowie Kommunikationsmöglichkeiten und Anschlussfähigkeit über die etablierten Grenzen einzelner Fachdisziplinen hinweg zu eröffnen. ${ }^{6}$ Im Rahmen dieser sich auch wissenschaftsorganisatorisch zunehmend etablierenden Kulturwissenschaften nehmen die Begriffe „Identität“" und „Memoria“" eine führende Stellung ein. Sichtbar wird dies nicht zuletzt in der jüngst erschienenen Einführungs- und Handbuchliteratur, die erste Standortbestimmungen innerhalb dieses sich noch formierenden Ansatzes der Kulturwissenschaften vornimmt und „Erinnerung“ bzw. „Gedächtnis“ und „Identität“ als einige ihrer bevorzugten Untersuchungsgegenstände ausweist. ${ }^{\text {" }}$

Es ist nicht leicht, auf einem so pulsierenden und unüberschaubaren, zugleich von unterschiedlichen fachdisziplinären Voraussetzungen und konkurrierenden Deutungshoheiten geprägten Feld dem Verdikt zu entgehen, man produziere durch die Rede von „Erinnerung“ und „kollektiver Identität“ nur auf den Status von „Plastikwörtern“ reduzierte Phrasen eines aktuellen, vom Zeitgeist bestimmten Wissenschaftsjargons. ${ }^{10} \mathrm{Im}$ folgenden soll daher der in dieser Studie verfolgte methodische Ansatz durch eine Bezugnahme auf einschlägige Theoreme und Forschungsfelder der Erinnerungsforschung präzisiert werden. Dabei geht es weniger darum, eine umfassende Bestandsaufnahme der unterschiedlichen Richtungen kulturwissenschaftlicher Erinnerungsforschung zu bieten. Vielmehr sollen gezielt diejenigen Ansatzpunkte - und Leerstellen - hervortreten, die in der vorliegenden Studie aufgenommen und weiterentwickelt werden. Von zentraler Bedeutung sind dabei zwei prominente Forschungsrichtungen, die unterschiedliche und bis in die jüngste Gegenwart hinein voneinander unabhängig operierende Zugänge zur Memoriathematik entwickelt haben: der vor allem von Jan und Aleida Assmann begründete, um das „kulturelle Gedächtnis“ als zentrale Kategorie der

${ }_{6} \quad$ Exemplarisch für das Konzept der neuen Kulturgeschichte, die nicht mehr als ein Segment der Geschichtswissenschaft, sondern als Öffnung der Geschichtswissenschaft hin auf andere Bereiche der Kulturwissenschaften verstanden wird, ist Daniel 2001, bes. 7-25.

$7 \quad$ Vgl. A. Assmann/Friese 1998, 11.

8 Zur Memoria als kulturwissenschaftlichem Leitparadigma der Gegenwart vgl. J. Assmann ${ }^{21997,}$ 11; Große-Kracht 1996, 21f.; Borsdorf/Grütter 1999, 1-3; A. Assmann 2003.

9 Vgl. z. B. Landwehr/Stockhorst 2004, 193-214 („Identität und Alterität“), 240-263 („Wahrnehmung und Gedächtnis“). Das 2004 erschienene „Handbuch der Kulturwissenschaften“ führt „Identität“ und „Geschichte“ (unter Einschluss von „Geschichtsbewusstsein, Erinnerung und Gedächtnis als fundamentalen Medien und Modi der kulturelle(n) Überlieferung“ [Jaeger/Liebsch 2004, XII]) als Schlüsselbegriffe der Kulturwissenschaften auf (Handbuch der Kulturwissenschaften, Bd. 1: Grundlagen und Schlüsselbegriffe, hrsg. von Friedrich Jaeger/Burkhard Liebsch, Stuttgart/Weimar 2004, 277-363, 365-531).

10 So Niethammer 2000, bes. 9-70, der damit die Verwendung des Begriffs ,kollektive Identität“ in - vorzugsweise nichtwissenschaftlichen - Diskursen der Gegenwart in den Blick nimmt und die massenhafte Produktion unterschiedlicher Identitätsdiskurse mit ihrer pseudowissenschaftlichen Terminologie und damit einhergehenden Verkürzungen und Vereinfachungen einer ideologiekritischen Bewertung unterzieht. Skeptisch hinsichtlich des innovativen Potentials kulturwissenschaftlicher Vernetzung unter Leitbegriffen wie „Erinnerung“ ist Walter 2004, 24. 
Identitätsstiftung kreisende Ansatz, der „Erinnerung“ als eine für die Formierung und Entwicklung von „Kultur“ zentrale Kategorie auffasst, und die mediävistische Memoriaforschung, die sich unter der Ägide von Otto Gerhard Oexle und Michael Borgolte zu einem maßgeblichen Forschungsschwerpunkt in der Mediävistik entwickelt hat und „Erinnerung“ in ähnlicher Weise als ein umfassendes, die politischen, gesellschaftlichen und kulturellen Strukturen gleichermaßen prägendes Phänomen begreift. Die vorliegende Studie zu den stadtrömischen Erinnerungsräumen spätantiker Heiligenmemoria verfolgt das Ziel, sich gleichsam auf der Schnittstelle zwischen diesen beiden Forschungsrichtungen zu bewegen, die trotz der eng miteinander verwandten Anliegen bis in die jüngste Gegenwart hinein ihre eigenen Wege gegangen sind. Diese Verortung zwischen der kulturwissenschaftlichen und der mediävistischen Erinnerungsforschung ermöglicht es, die heuristischen Stärken der jeweiligen Ansätze miteinander zu verbinden und für die Frage nach der stadtrömischen Heiligenerinnerung in der Spätantike fruchtbar zu machen. Neben den Vorzügen sollen jedoch gleichzeitig auch die Begrenzungen dieser beiden Richtungen verdeutlicht werden, die auf der einen Seite in konzeptionellen Leerstellen (der Relation von kommunikativem und kulturellem Gedächtnis), auf der anderen Seite in einer unzureichenden Differenzierung des Erinnerungsparadigmas (Memoria als Gabentausch) bestehen. In diesem Versuch, unterschiedliche Ansätze der Erinnerungsforschung anhand der Thematik des spätantiken Heiligenkults in Rom zusammenzuführen und weiter zu entwickeln, liegt - jenseits einer nicht weiter begründbaren Faszination, die sein Untersuchungsgegenstand auf den Verfasser und, wie er hofft, auch den Leser ausübt - ein wesentliches Motiv und Anliegen dieses Buches. Es versteht sich daher nicht nur als eine Fallstudie historischer Erinnerungsforschung in einem historischen Bereich, in dem dieses Konzept bisher noch nicht zur Anwendung gekommen ist, sondern auch als ein konstruktiver Beitrag zur Weiterentwicklung des Erinnerungskonzepts selbst und seiner Verwendung als kulturwissenschaftliches Forschungsparadigma.

\section{1. 1. Kulturwissenschaftliche und mediävistische Erinnerungsforschung}

Trotz - oder auch: wegen - der explosionsartigen Ausweitung von Studien zur Erinnerung gibt es derzeit keine umfassende Theorie, die einen Anspruch darauf erheben könnte, die komplexen Phänomene der Genese, Veränderung und identitätsstiftenden Wirkung von Erinnerung systematisch zu erfassen und in ein kohärentes Modell zu bringen. ${ }^{11}$ Dennoch haben bestimmte An-

11 Vgl. dazu A. Assmann 1999, 16, Eggers 2003, 220 und zuletzt die Bemerkung von Patrick Krassnitzer (Rezension zu Welzer 2002, in: HSozKult vom 26. 8. 2003), dass die von ihm rezensierte 
sätze die kulturwissenschaftliche Erinnerungsforschung der letzten Jahre maßgeblich geprägt und Ergebnisse erarbeitet, die auch die gegenwärtige Forschungsdiskussion um Erinnerung und kollektive Identitätsstiftung bestimmen. Hervorzuheben sind insbesondere drei Richtungen, die in etwa zeitgleich, jedoch unabhängig voneinander und mit unterschiedlichem methodischen Zuschnitt die Thematik „Erinnerung und kollektive Identität“ aufgeworfen haben. Seit den ausgehenden 1980er Jahren haben in Deutschland vor allem Jan und Aleida Assmann in Weiterführung älterer Ansätze von Aby Warburg und Maurice Halbwachs die Funktionen eines ,kulturellen Gedächtnisses“, seine Genese, Erscheinungsformen und Transformationen sowohl theoretisch als auch in Fallstudien zur identitätsstiftenden Funktion von Erinnerung für Gesellschaften untersucht. ${ }^{12}$ In denselben Zeitraum fallen die Anfänge der Diskussion eines „sozialen Gedächtnisses“ (social memory), die u. a. von Peter Burke angestoßen wurde und gegenwärtig vor allem die angloamerikanische Wissenschaftslandschaft prägt. ${ }^{13}$ Weniger an analytischen Fragen als an der praktischen Schaffung eines nationalen Gedächtnisses interessiert, hat in Frankreich Pierre Nora sein Konzept der „lieux de mémoire“ entwickelt, das zum Vorbild für ähnliche Projekte in einer Reihe von weiteren Ländern wurde und - unter veränderten Vorzeichen - vor kurzem auch in Deutschland aufgegriffen worden ist. ${ }^{14}$ Unter diesen Ansätzen kann am

Arbeit trotz ihrer weiterführenden Ansätze den in Aussicht gestellten Anspruch einer „kohärenten Erinnerungstheorie fast erwartungsgemäß (!) nicht wirklich erfüll[e]““.

12 Hier sei, insbesondere für die Verbindung von theoretischer Konzeptionalisierung und ihrer praktischen Applikation auf historische Gesellschaften, auf die Studie von J. Assmann ${ }^{2} 1997$ verwiesen; eine Zusammenfassung seiner Konzeption des „kulturellen Gedächtnisses“ bietet J. Assmann 1988. Im Unterschied dazu verzichtet A. Assmann 1999 explizit darauf, eine systematische Theorie des Gedächtnisses vorzulegen (ebd., 16), sondern lotet eher die Bandbreite der von der Gedächtnisthematik berührten Aspekte aus. Ihre „Erinnerungsräume“ konzentrieren sich weniger auf die wechselseitigen Beziehungen zwischen den sozialen Prozessen und den durch sie bedingten und auf sie zurückwirkenden Formen der Gedächtnisbildung, als vielmehr auf die unterschiedlichen Strukturen, Erscheinungsformen und Medien des Gedächtnisses.

13 Vgl. Burke 1991 (1989); Connerton 1989, 6-40; Alcock/van Dyke 2003, 1-3. Im Unterschied zum kulturellen Gedächtnis, das monolithische Züge trägt, stellt die social memory-Forschung stärker die konkurrierenden und konfligierenden Erinnerungen unterschiedlicher Gruppen in den Vordergrund, die durch Geschlecht, sozialen Status, Religion, Alter oder ähnliche Faktoren bestimmt sind; vgl. dazu auch u., S. 9f.

14 Für erste Überlegungen zur „mémoire collective“ als Forschungsparadigma s. Nora 1978; programmatischen Charakter hat die Einleitung zum ersten Band der „Lieux de mémoire“ (deutsch Nora 1990). Nora entwirft die Gegenwartssituation einer ihrer lebendigen Traditionen entfremdeten französischen Nation, der allerdings noch ihr Wissen um (und die Verpflichtung auf) ihre Traditionen verblieben sei. Diese kristallisieren sich in den von Nora so bezeichneten „Erinnerungsorten“ („lieux de mémoire“), deren Erinnerungsinhalte früher in einem „,milieu de mémoire" als unreflektiertes Wissen traditionell weitergegeben und erst mit dem Verlust der Tradition zu „lieux de mémoire“ objektiviert worden seien. In Deutschland ist das Konzept der „lieux de mémoire“ vor einigen Jahren ebenfalls aufgegriffen worden, allerdings ohne den problematischen - normativen Anspruch, durch Erinnerungsorte Identität stiften zu wollen (vgl. 
ehesten die mittlerweile klassische Studie von Jan Assmann zum kulturellen Gedächtnis als ein systematischer Versuch gelten, das Phänomen der Erinnerung in seiner gesamtgesellschaftlich-kulturellen Bedeutung zu erfassen und auf diese Weise ein umfassendes Modell von Erinnerung, ihren unterschiedlichen Feldern und Medien sowie deren Auswirkung auf Formen der kollektiven Identitätsstiftung zu präsentieren. Sie soll daher als Ausgangspunkt für die folgenden Bemerkungen zur kulturwissenschaftlichen Erinnerungsforschung dienen.

Die Grundlage für Assmanns Ausführungen bildet die These von der sozialen Struktur des Gedächtnisses und seiner identitätsstiftenden Funktion. Die Gegenstände der Erinnerung sind - durch Kommunikation und Interaktion - sozial generiert und demgemäß gruppenspezifische Hervorbringungen. Das Gedächtnis umfasst auf diese Weise einen Fundus gemeinsamer Vorstellungen, die für eine Gruppe spezifisch sind und in der Abgrenzung nach außen die Formierung einer Identität erlauben. In unmittelbarer Nachbarschaft zu wissenssoziologischen Ansätzen erscheint Erinnerung damit als Bereich eines kollektiv geteilten „Wissens“ überhaupt, das gesellschaftlich vermittelte Erfahrungswerte erzeugt, die der Orientierung und der Erwartbarkeit von Handlungen dienen. ${ }^{15}$ Mit dieser kollektiven Eigenschaft des Gedächtnisses und seiner Funktion für die Identitätsstiftung hängt es zusammen, dass die Vergangenheitsbezüge der Erinnerung eine Reihe von Charakteristika aufweisen, die sie von denen der „Geschichte“ unterscheiden und die identitätsbildende Funktion von Erinnerung im Unterschied zur Geschichte herausstellen: Während das Gedächtnis historischen Wandel ausblendet und Kontinuitäten wahrnimmt, richtet die Geschichte umgekehrt ihr Augenmerk auf Veränderungen und Differenzen. Erinnerung stiftet im Wiedererkennen der eigenen Gegenwart in der Vergangenheit Identität, Geschichte hingegen betont die Distanz zwischen der Gegenwart und der Vergangenheit als „dem anderen“. Geschichtliche Vergangenheitswahrnehmung ist geprägt von Abstraktionen und Strukturen; dem steht auf seiten des Gedächtnisses dessen

die von Etienne François und Hagen Schulze herausgegebenen 3 Bände „Deutsche Erinnerungsorte“, München 2001; für die von Nora abweichende Konzeption s. François/Schulze 2001, 23f. u. W. Schulze 2003, 609). Instruktive Kritik an Noras pauschaler These vom „Verlust des Gedächtnisses“ als Voraussetzung für seine Objektivierung in Erinnerungsorten übt A. Assmann 1999, bes. 11-16, die zum einen den Begriff des Gedächtnisses präziser fasst (Differenzierung zwischen Lerngedächtnis, Bildungsgedächtnis und Erfahrungsgedächtnis) und darauf hinweist, dass das Entstehen spezifisch deutscher Erinnerungsorte nicht aus Traditionsverlust erklärt werden kann (ebd., 338f.; ähnliche Kritik bei Borsdorf/Grütter 1999, 4; Hölkeskamp 2001, 125f.). Wie im folgenden deutlich werden wird, hat der in dieser Arbeit verwendete Begriff des „Erinnerungsraums“ trotz der begrifflichen Nähe mit Noras Konzept der,„lieux de mémoire“ bzw. „Erinnerungsorte“ nichts zu tun, sondern ist auf topographisch präzise lokalisierbare Orte begrenzt (s. dazu u., S. 19-23).

15 Vgl. J. Assmann 1988, 11f. und dens. ${ }^{21997,47 f .}$ 
„Identitätskonkretheit“ als Charakteristikum und identitätsstiftendes Moment gegenüber. ${ }^{16}$

Über diese - im wesentlichen bereits von Maurice Halbwachs in den 20er und 30er Jahren angestellten - Beobachtungen zu Funktionsweise und Struktur des Gedächtnisses hinaus hat Jan Assmann vor allem die Erscheinungsformen des kollektiven Gedächtnisses präzisiert, zum anderen die Mechanismen zur dauerhaften Sicherung und Verstetigung dieses Gedächtnisses untersucht. Die von Assmann kategorisierten Bereiche „mimetisches Gedächtnis“ (Nachahmung von Handlungen), „Dinggedächtnis“ und „Sprache und Kommunikation" sind insofern ephemer, vom Vergessen bedroht, als sie Gegenstand der Alltagskommunikation und über eine pragmatische Funktion hinaus nicht mit dauerhaftem Sinn und Bedeutung belegt sind. Ihre wirksame Immunisierung gegen das Vergessen erfolgt erst durch kulturelle Festschreibung, durch das, was Assmann als das „kulturelle Gedächtnis“ bezeichnet und vom „kommunikativen Gedächtnis“ von Kollektiven unterscheidet: An die Stelle routinehafter Handlungen treten rituelle Praktiken, rein zweckbezogene Gegenstände entwickeln sich zu Repräsentationen, Symbolen und Denkmälern, aus der verbalen Alltagskommunikation werden zeremonielle Kommunikationsformen sowie sinnspeichernde und -zirkulierende Texte ausgelagert. ${ }^{17}$ Die Differenz zwischen kulturellem und kommunikativem Gedächtnis schlägt sich außer in den Medien, Inhalten und Trägern der Erinnerung vor allem in der unterschiedlichen Zeitstruktur nieder. In Anlehnung an die Erkenntnisse der „Oral History“ geht Assmann von einem „floating gap“ aus, den er auf drei bis vier Generationen bzw. den Zeitraum von 80 bis 100 Jahren bemisst, und der den Zeithorizont des kommunikativen Gedächtnisses bezeichnet, der mit der fortschreitenden Gegenwart mitwandert. ${ }^{18}$ Jenseits dieses Zeitraums bleiben nur rituelle, dingliche und sprachlich-textuelle Objektivationen erinnerungsfähig, die in das kulturelle Gedächtnis aufgenommen werden und Erinnerungsfiguren bilden, die der Einweisung durch Spezialisten und Experten des kulturellen Gedächtnisses unterliegen. ${ }^{19}$ Assmanns Interesse gilt vor allem dem kulturellen Gedächtnis, in dem bestimmte Inhalte des kommunikativen Gedächtnisses ausgewählt, konserviert und kanonisiert

16 Vgl. J. Assmann 21997, $42-45$ (Referat der Positionen von Halbwachs); Nora 1990, 12f. spitzt diese Gegensätzlichkeit von Geschichte und Erinnerung zu einem regelrechten Antagonismus zu, in dem die Geschichtswissenschaft das Gedächtnis zerstöre. A. Assmann 1999, 130-145 schwächt hingegen zurecht die in der begrifflichen Trennung von „Geschichte“ und „Gedächtnis“ implizierte Opposition ab und plädiert stattdessen für eine Unterscheidung zwischen einem (identitätsbildenden) Funktions- und einem Speichergedächtnis, dessen Inhalte für unterschiedliche Funktionsgedächtnisse verfügbar gemacht werden können: Geschichte und Gedächtnis stehen in Verbindung mit-, nicht in Konkurrenz zueinander.

17 J. Assmann 21997, 20f.; vgl. auch dens., 1988, 12.

18 J. Assmann 21997, 48-53.

19 Zur Partizipationsstruktur des kulturellen Gedächtnisses s. ebd., 53-55. 
werden. Innerhalb dieses kulturellen Gedächtnisses wiederum wendet Assmann seine Aufmerksamkeit besonders den textuellen Erinnerungsfiguren zu: Die Entwicklung der Schrift und der damit verbundene Übergang von ,ritueller“ zu „textueller Kohärenz“, die literarische Kanonbildung und die kommentierende Interpretation kanonisierter Texte stehen im Zentrum seiner Überlegungen. Demgegenüber nehmen die rituellen und dinglich-monumentalen Ausprägungen des kulturellen Gedächtnisses bei ihm nur einen vergleichsweise geringen Raum ein. ${ }^{20}$

Assmanns Modell stellt eine Reihe von grundlegenden Kategorien bereit, die einen heuristisch fruchtbaren Interpretationsrahmen abstecken. Dennoch beinhaltet sein Konzept auch Leerstellen und Unstimmigkeiten, die im Zuge der kulturwissenschaftlichen Erinnerungsforschung der letzten Jahre mit zunehmender Deutlichkeit hervorgetreten sind. Ergänzungs- und Korrekturbedarf zeichnet sich vor allem in zwei Punkten ab: in der Relation von kommunikativem und kulturellem Gedächtnis sowie in der Beziehbarkeit des kulturellen Gedächtnisses auf die Identitätsstiftung von Kollektiven und Gruppen. Beide Kritikpunkte sind wesentlich dadurch bedingt, dass Assmann um den Begriff des Gedächtnisses eine regelrechte Kulturtheorie organisiert und Erinnerung als eine umfassende Kategorie zur Produktion von kulturellem Sinn begreift. ${ }^{21}$ Diesem weitreichenden Erklärungsanspruch, der Gedenken und Erinnern zum Motor kultureller Entwicklung macht, entspricht jedoch nur eine vergleichsweise unscharfe Vorstellung dessen, was der Untersuchungsgegenstand eines sozial generierten Gedächtnisses sein soll, welchen Entstehungsbedingungen seine Produktion unterliegt und in welcher Weise dieses Gedächtnis identitätsstiftende Funktion entfaltet. Zwar betont Assmann den engen Zusammenhang von kommunikativem und kulturellem Gedächtnis, indem er die „konnektive Struktur“ einer Kultur als Zusammenspiel eben dieser beiden Erinnerungsmodi begreift: Kommunikatives und kulturelles Gedächtnis bilden in Assmanns Terminologie die Sozial- und die Zeitdimension einer Kultur aus, in der synchrone und diachrone Kommunikation zusammenwirken, um einen Haushalt an kognitiven und praxeologischen Deutungs- und Orientierungsleistungen zu begründen. ${ }^{22}$ Die notwen-

$20 \quad$ Ebd. 17f.; 87-129. Eine andere Akzentsetzung bei Hölkeskamp 2001; Flaig 2003, 49-98, die mit Blick auf das republikanische Rom die Bedeutung der Vernetzung von unterschiedlichen Formen des kulturellen Gedächtnisses (Riten, Orte, literarisch vermittelte exempla) hervorheben.

21 Dieser kulturtheoretische Anspruch des Erinnerungsparadigmas wird besonders deutlich von J. Assmann 21997, 24f., 46f. vorgetragen.

22 Zur „konnektiven Struktur“ einer Kultur, in der ein spezifisch vergangenheitsbezogenes Wissen (,Zeitdimension“) als Teil eines weiter gefassten Bereichs gemeinsamer Regeln und Werte („Sozialdimension") erscheint, der in Anlehnung an wissenssoziologische Ansätze konzeptualisiert wird, vgl. J. Assmann 21997, 16f., 48. Für die Gleichsetzung dieser beiden Dimensionen von Kultur mit dem kulturellen bzw. kommunikativen Gedächtnis vgl. dens. 2000, 19. In ähnlicher 
dige Schlussfolgerung, dass kommunikatives und kulturelles Gedächtnis unmittelbar zusammengehören und sich allenfalls analytisch voneinander trennen lassen, ${ }^{23}$ wird allerdings nicht hinreichend berücksichtigt. So blendet Assmann durch die Konzentration auf das kulturelle Gedächtnis die Frage nach den Schnittstellen und nach den Übergängen, die zwischen diesen beiden modi memorandi bestehen, weitgehend aus. Dies ist zwar insofern nachvollziehbar, als die Beschäftigung mit dem kommunikativen Gedächtnis in methodischer Hinsicht andere Annäherungen an den Gegenstand verlangt, die auch neurobiologische und entwicklungspsychologische Momente berücksichtigen. ${ }^{24}$ Dennoch geraten auf diese Weise wesentliche Aspekte, die die soziale Generierung von Erinnerung und deren formative Bedeutung für die Identität von Kollektiven betreffen, aus dem Blick. Welche Gruppen an der Produktion des kulturellen Gedächtnisses beteiligt sind und wie es zur Entstehung einer Erinnerungskultur kommt, sind Vorgänge, die sich mit Assmanns Modell nur unzureichend erfassen lassen. Indem Assmann den Schwerpunkt auf die Verstetigung, Reproduktion und Sicherung des kulturellen Gedächtnisses legt, rücken diejenigen Prozesse an den Rand der Betrachtung, die zur Ausbildung dieses Gedächtnisses führen und die in zahlreichen Gesellschaften auf der Ebene des kommunikativen Gedächtnisses anzusiedeln sind. ${ }^{25}$ Sein Modell ist damit im wesentlichen statisch angelegt und wenig geeignet, den historischen Wandel und das Veränderungspotential von gruppenbezogener Erinnerung in den Blick zu nehmen: Die Zusammenhänge zwischen gesellschaftlichen Veränderungen, sich wandelnden Sozialbeziehungen innerhalb von Gruppen und den Folgen, die diese Vorgänge für die Formierung von kollektiven Gedächtnissen haben, bleiben durch die einseitige Konzentration auf das kulturelle Gedächtnis tendenziell unberücksichtigt.

An diese Beobachtung, dass für Prozesse kollektiver Identitätsstiftung dem kommunikativen Gedächtnis eine wesentliche Bedeutung im Rahmen kollektiven Erinnerns zukommt, knüpft sich die verwandte Frage, ob das Konzept eines kulturellen Gedächtnisses geeignet ist, Formen kollektiver Identitätsstiftung in historischen Gesellschaften adäquat zu erfassen. ${ }^{26}$ Jan

Weise verwendet Walter 2004, 18-20 den Begriff der „Geschichtskultur“, um das „synchrone und diachrone soziale Gedächtnis eines Kollektivs“ (ebd., 20) zu bezeichnen.

23 Zurecht hervorgehoben von Welzer 2002, 15.

$24 \quad$ Vgl. dazu insgesamt Welzer 2002.

25 Dies hat zuletzt die Studie von Walter 2004 zur republikanischen Erinnerungskultur im antiken Rom deutlich gemacht: Walter weist überzeugend nach, dass die im Begriff und Konzept des mos maiorum verkörperten Inhalte handlungsleitender Erinnerung keine starre Größe darstellten, sondern ein dynamisches und wandelbares Feld bildeten, auf das die unterschiedlichen gentes Einfluss nahmen, um politisches und soziales Prestige zu sichern.

26 Auch Assmann selbst ist sich dieses Problems bewusst, hält jedoch die Ausweitung des Gedächtnisbegriffes von eng umrissenen Gruppen auf komplexe Gesellschaften und Kulturen für möglich (vgl. J. Assmann ${ }^{21997, ~ 46 f .) . ~}$ 
Assmann hat sein Konzept des kulturellen Gedächtnisses unter maßgeblicher Bezugnahme auf die Fallstudien Ägypten und Israel entwickelt, die jedoch insofern als historische Sonderfälle gelten können, als in ihnen spezifische politische und religiöse Voraussetzungen herrschten, die der Ausbildung eines monolithischen kulturellen Gedächtnisses förderlich waren. Auf das antike Griechenland und die Zeit der römischen Republik sind diese Verhältnisse hingegen nicht übertragbar: Sowohl in der griechischen Poliswelt mit ihrer Vielfalt an mythologischen Gründungserzählungen als auch in der politischen Kultur des republikanischen Rom mit ihren konkurrierenden gentilizischen Erinnerungen formierten sich in starkem Maße Partikulargedächtnisse, die keiner zentralen Steuerung und Kontrolle unterlagen und für die das Konzept eines einheitlichen kulturellen Gedächtnisses nicht angemessen erscheint. ${ }^{27}$ Insofern ist bedenkenswert, ob nicht der holistische Begriff der Kultur eine Kohäsion und Integrationskraft suggeriert, unter der die rivalisierenden und multiplen Gedächtnisse von Gruppen und Kollektiven zu verschwinden drohen und ob die Frage nach der identitätsstiftenden Wirkung von Erinnerung die Aufmerksamkeit nicht stärker auf die kommunikativen Prozesse lenken sollte, die der Formierung dieser kollektiven Identitäten zugrunde liegen. ${ }^{28}$

Als Bilanz aus dem Bisherigen lässt sich festhalten, dass das kulturwissenschaftliche Erinnerungsparadigma einer stärkeren Konzentration auf das kommunikative Gedächtnis und auf einzelne Kollektive als Träger der Erinnerung bedarf, um den elementaren Zusammenhang von Erinnerung und kollektiver Identitätsstiftung zu analysieren. Die Anwendbarkeit dieses Postulats auf historische Fallstudien begegnet zugegebenermaßen nicht unerheblichen methodischen Schwierigkeiten, die durch die Quellenlage bedingt sind. Beobachtungen zum kommunikativen Gedächtnis fallen in den Bereich der „Oral History“ und der neurobiologischen und autobiographischen Gedächtnisforschung, die mit Blick auf die vergleichsweise spärlich fließenden antiken Quellen zwar als ein quellenkritisches Korrektiv dienen können, ${ }^{29}$ deren Funktionsweise jedoch in der Regel nicht aus diesem Quellenbestand heraus entwickelt werden kann. Auch die komplexen Mechanismen der Identitätsstiftung in vergangenen Gesellschaften sind aufgrund der unzureichen-

$27 \quad$ Hervorgehoben von Walter 2004, bes. 24f., Jung 2006, $18 \mathrm{f}$.

28 In diesem Sinne auch Burke 1991 (1989), 298. Eine vergleichbare Kritik ist in den letzten Jahren auch am Begriff der „Mentalität“ formuliert worden, der - ähnlich wie das „kulturelle Gedächtnis“ - mit der vorgängigen (und suggestiven) Vorstellung eines hohen Grades an gesellschaftlicher Homogenität operiert und dazu tendiert, Differenzierungen nach unterschiedlichen Gruppen auszublenden; vgl. dazu Gotter 2001, 269, der für eine gleichsam induktive und handlungsorientierte Verwendung des Mentalitätsbegriffs - Rückschlüsse vom Verhalten historischer Akteure auf deren Mentalitäten anstelle einer apriorischen Annahme einer kollektiven Mentalität - plädiert.

29 Zu den Konsequenzen der „Oral History“ und biologischen Gedächtnisforschung für die antike und mittelalterliche Quellenkritik vgl. zuletzt Fried 2004, bes. 372-380. 
den Quellenlage nur in Ansätzen zu erfassen. Dass Erinnerung auf personaler ebenso wie auf kollektiver Ebene eine identitätsstiftende Funktion hat, dürfte zwar als eine petitio principi kaum zu bezweifeln sein: ${ }^{30}$ In dem Maße, in dem das Gedächtnis im Unterschied zur Geschichte dazu tendiert, Differenzen auszublenden und Kontinuitäten zu betonen, ${ }^{31}$ nimmt es wesentliche Integrationsleistungen vor, die für eine gelungene Identitätsstiftung konstitutiv sind. ${ }^{32}$ Wesentlich schwieriger erscheint es jedoch, diese Vorgänge der kollektiven Identitätsstiftung in ihren Ausbildungen historisch zu greifen. In dieser Hinsicht haben insbesondere die Arbeiten von Pierre Bourdieu das Verständnis dafür geschärft, einerseits zwischen professionell-wissenschaftlichen kollektiven Identitätskonstruktionen, die simplifizierend von Klassen und Gruppen als den Objekten und Trägern sozialen Handelns reden, und andererseits den Prozessen einer praxeologisch vermittelten Identitätsbildung, die quer zu diesen professionellen Taxonomien liegen, zu differenzieren. Bourdieu konzeptualisiert die soziale Realität als einen Raum, in dem die Akteure nicht durch die Zugehörigkeit zu einer umrissenen Gruppe, sondern durch Relationen zueinander bestimmt werden, die auf unterschiedlichen Feldern sozialen Handelns wirksam sind und ihre Positionen innerhalb des sozialen Raums festlegen. ${ }^{33}$ Dass Inhaber analoger Positionen sich auch zu Gruppen zusammenfinden, ist zwar wahrscheinlich, doch identitätsbestimmend sind in erster Linie die Verortungen in den unterschiedlichen Feldern des sozialen Raums. Nach Bourdieu ist es die handlungspraktische Einnahme dieser Positionen, die den Akteuren einen Ort innerhalb der Kräfteverhältnisse zuweist und - damit wechselseitig untrennbar verbunden auch die Wahrnehmungen vermittelt, die stabilisierend und modifizierend auf die Kräfteverhältnisse einwirkt. Dem Handeln und Sprechen der Akteure wohnt demnach eine eigene Logik inne, die sich in der sozialen Praxis - trotz

30 Der elementare Zusammenhang zwischen Erinnerung und Identität wird von den unterschiedlichen Richtungen der historischen Erinnerungsforschung durchgängig thematisiert (vgl. z. B. Nora 1990, bes. S. 12f.; Oexle 1995, 10; J. Assmann 21997, 16f.; A. Assmann 1999, 130-133).

31 Vgl. o., S. 6f.

32 Vgl. Straub 1998, bes. 75, der in der Fähigkeit, ,Kontinuität und Kohärenz angesichts diachroner und synchroner Differenzerfahrungen“ zu bilden, den gemeinsamen Nenner aller Definitionen des personalen Identitätsbegriffs erkennt. Prinzipiell ist dieses personale Identitätskonzept auch auf Kollektive übertragbar: In beiden Fällen ist Identität an Integrationsleitungen gebunden, die den Konstruktcharakter, der dem Identitätsbegriff inhärent ist, deutlich hervortreten lassen und ihm damit jegliche essentialistische Grundlage entziehen. Straub betont allerdings die Notwendigkeit, zwischen „,normierenden“ und ,rekonstruktiven“ Typen kollektiver Identitätsstiftung zu unterscheiden: Während erstere rein auf äußeren Zuschreibungen beruhen, die häufig ohne Rücksicht auf die Lebens- und Erfahrungswelt der „,betroffenen“ Akteure erfolgen, stellen sich die letzteren als das Ergebnis kollektiver Übereinkünfte und Aushandlungen dar, die meist unbewusst vorgenommen werden (vgl. ebd., 96-104 und die folgenden Bemerkungen zu Bourdieu).

33 Zum sozialen Raum und seinen unterschiedlichen Feldern (ökonomisches, kulturelles, soziales Feld mit weiteren Ableitungen) vgl. Bourdieu 1985, 9-15. 
und gegen die Taxonomien und Identitätskonstruktionen, die von professionellen Verwaltern der symbolischen Gewalt entworfen werden - reproduziert und dabei gleichzeitig modifiziert. ${ }^{34}$

Im Unterschied zur Soziologie ${ }^{35}$ kann es aus althistorischer Perspektive in Anbetracht einer fragmentarischen Quellensituation, die in Ermangelung serieller Daten nur selektive Einblicke in Handlungssituationen und -kontexte erlaubt, weder darum gehen, eine Vermessung des sozialen Raums vorzunehmen, noch darum, einen eigenständigen Beitrag zur „Oral-History“ zu leisten. Vielmehr gilt es, die Erkenntnisse zum kommunikativen Gedächtnis und zu Prozessen kollektiver Identitätsstiftung umzusetzen und bei der Anwendung des Erinnerungsparadigmas zu berücksichtigen. Eine derartige Orientierung läuft darauf hinaus, sich nicht auf die Frage nach der konstitutiven Bedeutung von Erinnerung für die Erzeugung kultureller Wert- und Symbolsysteme zu beschränken und stattdessen das Augenmerk auf die kommunikativen und interaktiven Praktiken des Erinnerns selbst zu richten. Auf diese Weise geraten verstärkt diejenigen Prozesse in den Blick, die unterhalb des kulturellen Gedächtnisses angesiedelt und im vorigen als die wesentlichen Leerstellen angesprochen worden sind, in der dieses Modell der Ergänzung bedarf: ${ }^{36}$ Sowohl die Erscheinungsformen des kommunikativen Gedächtnisses und die Übergänge vom kommunikativen in das kulturelle Gedächtnis als auch die Differenzierung zwischen unterschiedlichen Erinnerungen von Kollektiven sowie der Zusammenhang zwischen kollektiver Erinnerung und praxeologisch vermittelter Identitätsstiftung sind nur dann adäquat zu erfassen, wenn man sich auf die Memoria als eine Hervorbringung sprechender und handelnder Akteure konzentriert und auf diese Weise der kulturtheoretischen Leitkategorie Erinnerung eine stärker auf einzelne Fallstudien ausgerichtete Akzentuierung gibt.

Einen derartigen Weg hat in den vergangenen Jahren vor allem die mediävistische Memoriaforschung beschritten. Anders als die kulturwissenschaftliche Erinnerungsforschung hat sie ihren Ausgang von einem konkreten Phänomen des Erinnerns, dem frühmittelalterlichen Totengedenken, genommen. Die Grundlage der Forschungen, deren Anfänge sich besonders mit den Na-

34 Bourdieu hat diese Einsicht aus der Kritik an einer reduktionistischen - da deren komplexe Verortungen innerhalb eines sozialen Raums nicht berücksichtigenden - Objektivierung von Klassen und Gruppen entwickelt, die sich in gleicher Weise gegen staatliche Institutionen, die marxistische Theorie und das akademische und intellektuelle Establishment in Frankreich richtet (vgl. hierzu und zum Postulat einer ihre eigenen Voraussetzungen reflektierenden Soziologie exemplarisch dens. 1985, 23-30 und 49-81).

35 Zum Programm einer auf statistischen Daten basierenden Analyse des sozialen Raums vgl. Bourdieu 1985, 12f.; für dessen praktische Umsetzung vgl. dens. 1982.

36 S. o., S. 9 f. 
men Karl Schmid und Joachim Wollasch verbinden, ${ }^{37}$ bildeten zunächst die zwischen den Vorstehern von Klöstern und Klerikergemeinschaften geschlossenen Gebetsverbrüderungen, die das gegenseitige Gedenken der verstorbenen Mitglieder - vor allem der Leiter - dieser Gemeinschaften sichern sollten. Diese kommemorialen Verbrüderungsformen, die sich bis in die Mitte des 8. Jh. zurückverfolgen lassen ${ }^{38}$ und historisch nachweisbare Vorläufer in entsprechenden Vereinbarungen zwischen Klöstern und einzelnen Stiftern und Wohltätern haben, ${ }^{39}$ ließen den Zusammenhang von Erinnerungspraktiken und kollektiver Identitätsstiftung unmittelbar hervortreten. Zunächst galt das Interesse jedoch weniger dieser Verbindung als dem sozialgeschichtlichen Quellenwert der Verbrüderungsbücher (libri memoriales) und Totenbücher (Nekrologien): ${ }^{40}$ Das in ihnen enthaltene umfangreiche Namensmaterial lieferte Aufschlüsse für prosopographische Fragen nach der Verbindung zwischen einzelnen Klöstern sowie deren Beziehungen zu Königtum und Adel. ${ }^{41}$ Erst seit den Arbeiten von Otto G. Oexle rückte die Bedeutung des Gedenkens als soziale Praxis und die formative Bedeutung dieses Phänomens für die Bildung von Kollektiven stärker in den Vordergrund. Oexle begriff - wie vor ihm bereits Schmid - das Gedenken unter Bezugnahme auf Marcel Mauss als Bestandteil eines Gabentauschs, bei dem das Gebetsgedenken als spirituelle Gegengabe für empfangene materielle Leistungen fungierte. ${ }^{42}$ Die Voraussetzung dafür, dass das Totengedenken in dieser Weise wirksam werden konnte, lag in der Vorstellung begründet, dass Lebende und Tote über die Grenze des Todes hinweg durch einen reziproken

37 Die ersten Anstöße gingen von Gerd Tellenbach aus, der in den 1950er Jahren auf die Bedeutung der memorialgeschichtlichen Überlieferung für die Erforschung des Adels und der Klostergemeinschaften im früheren Mittelalter und der Reformzeit aufmerksam machte, und dessen Anregungen von Karl Schmid und Joachim Wollasch aufgenommen und zu umfassenden Forschungsprojekten weiterentwickelt wurden. Einen informativen Forschungsüberblick über die Entwicklung der Memoria-Forschung in der deutschen Mediävistik bietet Borgolte 1998.

38 Zu den frühesten Beispielen zählen die Gebetsbünde von Attigny (vermutlich 762) und Dingolfing (770), deren Gedenkbücher in die Reichenauer Memorialüberlieferung eingegangen sind (vgl. dazu Schmid/Oexle 1974; zur Entfaltung der Verbrüderungsbewegung seit der Mitte des 8. Jh. s. ebd., 94f.).

39 Für das Gebetsgedenken von Stiftern und Wohltätern vgl. die immer noch grundlegende Zusammenstellung bei Ebner 1890, 20-27, die das Material bis in die erste Hälfte des 6. Jh. zurückverfolgt.

40 Die mit den Gebetsbünden des 8. Jh. entstandenen libri memoriales, die summarische Auflistungen der beteiligten Personen enthielten, wurden seit dem 10. Jh. durch Nekrologien abgelöst, in denen die Kommemorierten auf kalendarische Tagesdaten verteilt wurden und die damit ein individuelles Gedenken ermöglichten. Zu den beiden Gattungen und ihrer inneren Zusammengehörigkeit als unterschiedliche Ausprägungen liturgischer Memorialliteratur vgl. Schmid/Wollasch 1967.

41 Zur prosopographischen und sozialgeschichtlichen Ausrichtung der Tellenbach-Schule vgl. Borgolte 1998, 199-203; dens. 2005, 23.

42 Vgl. Schmid/Oexle 1974, 73f.; Oexle 1976, 87f. 
Handlungszusammenhang miteinander verbunden blieben, der die Toten als einen gegenwärtigen und handlungsfähigen Teil in die Gemeinschaft der Lebenden mit einschloss. ${ }^{43}$ Auf diese Weise fungierte das Gebetsgedenken für die Toten als eine im engeren Sinne soziale Gabe, die sich an Mitglieder der Gemeinschaft richtete und diesen Handlungszusammenhang aktualisierte. Die Reziprozität dieser Beziehungen ist in den letzten Jahren vor allem mit Blick auf die mittelalterliche Stiftungspraxis herausgearbeitet worden. Wie insbesondere die Arbeiten von Michael Borgolte überzeugend gezeigt haben, beruhte die Perpetuierung des Stifterwillens in Ermangelung des Konzepts einer Stiftung als juristischer Person auf der Vorstellung, dass der Stifter als natürliche Person auch nach seinem Tod unter den Lebenden gegenwärtig blieb und als Rechtssubjekt die Aufrechterhaltung seiner Stiftung sicherte. ${ }^{44}$ Stiftergabe und Stiftergedenken bildeten auf diese Weise einen wechselseitigen Zusammenhang des Gebens und Nehmens, der ständig aktualisiert wurde und auf einem andauernden Kreislauf von Gabe und memorialer Gegengabe beruhte.

Mit seiner Bezugnahme auf Mauss hob Oexle nicht nur die strukturelle Bedeutung des Gebetsgedenkens für die Konstituierung von Gemeinschaften im Mittelalter hervor, sondern evozierte zugleich ein theoretisches Konzept, das die Ansätze für eine Ausdehnung der Erinnerung auf die unterschiedlichsten Bereiche der mittelalterlichen Kultur in sich trug. Bekanntlich hat Mauss mit Blick auf die von ihm untersuchten archaischen Gesellschaften nicht nur die soziale Bindungskraft des Gabentauschs hervorgehoben, sondern auch dessen Charakter als ein „totales gesellschaftliches Phänomen“ betont, das in intensiver Weise das gesamte gesellschaftliche und kulturelle Leben durchdrang: Gaben definierten den sozialen Status von Gebern und Empfängern, regelten rechtliche Vertragsbeziehungen, ökonomischen Austausch sowie religiöse Beziehungen zu den Göttern und bildeten den Anlass für die Produktion materieller Kulturgüter. ${ }^{45}$ In Anlehnung an dieses Modell hat Oexle der Memoria einen vergleichbaren Stellenwert für die mittelalterliche Kultur zuerkannt. ${ }^{46}$ Über die oben erwähnten Beispiele eines liturgisch bestimmten Gebetsgedenkens und seiner gattungsspezifischen Erscheinungsformen (libri memoriales, Nekrologien) hinaus weist er dem Totengedenken eine formative Bedeutung für zahlreiche weitere Quellengattungen und die in ihnen berührten Teilbereiche des gesellschaftlichen Lebens zu. Durch Oexles Beobachtung, dass ein kommemoratives Anliegen im Sinne ei-

43 Zur Gegenwart der Toten als geschäfts- und handlungsfähige Rechtssubjekte im sozialen Verkehr vgl. Oexle 1983, bes. 29f., 33f.; dens. 1985, 81.

44 Vgl. Borgolte 1988, bes. 83-94; zu den spezifischen religionsgeschichtlichen Voraussetzungen, auf denen diese Konzeption der mittelalterlichen Stiftung im lateinischen Westen beruht, vgl. zuletzt Lusiardi 2005, bes. 49-58.

45 Mauss 1968, bes. 17f., 88-90, 176-178.

46 Zur mittelalterlichen Memoria als „totalem Phänomen“ vgl. Oexle 1984, 394, ders. 1995, 39-48. 
ner Aufrechterhaltung des Gebetsgedenkens seinen Niederschlag auch in biographischen und hagiographischen, historiographischen und rechtssichernden Texten sowie repräsentativen Bauten und Bildern hinterlassen hat, ${ }^{47}$ avanciert die memoriale Gabe der Erinnerung zu einem umfassenden kulturellen Phänomen, das über das Totengedenken im engeren Sinne hinaus gesellschaftliche Handlungsbereiche und Institutionen mit ökonomischen, politischen und ästhetischen Implikationen erfasst. In Fortführung dieses Interpretationsansatzes hat zuletzt Michael Borgolte einen spezifischen Aspekt dieser Memorialbeziehungen - das mittelalterliche Stiftergedenken - untersucht und zum Ausgangspunkt für die Frage nach der Memoria als einem „totalen Phänomen“ der mittelalterlichen Kultur gemacht. ${ }^{48}$ Auf seine Initiative gehen auch die jüngsten Anstöße zurück, das Stiftungswesen unter kultur- und religionsvergleichender Perspektive zu untersuchen und damit die Übertragbarkeit des in der Mediävistik entwickelten Modells auf andere Kulturen zu prüfen. ${ }^{49}$

Obwohl die kulturwissenschaftliche und die mediävistische Erinnerungsforschung bis zum gegenwärtigen Zeitpunkt weitgehend unabhängig voneinander ihre Konzepte entwickelt und verfolgt haben, ${ }^{50}$ berühren sie sich nicht nur in der gemeinsamen Auffassung, dass Erinnerung eine kulturelle Leitkategorie bilde. Vielmehr stellen die beiden Forschungsrichtungen auch Ansätze bereit, die sich miteinander verbinden lassen und zur wechselseitigen Weiterentwicklung beitragen. So hat die mediävistische Memoriadiskussion - forschungsgeschichtlich bedingt - Zugänge zu dieser Thematik eröffnet, die an neuralgischen Punkten das Modell des kulturellen Gedächtnisses ergänzen. Die Studien zum Totengedenken und der Memoria als sozialer Praxis, von denen die mediävistische Memoriaforschung ihren Ausgang genommen hat, liefern zum einen Einblicke in den Zusammenhang von Memoria und handlungspraktisch vermittelter Identitätsstiftung, aus denen die formative Bedeutung des Erinnerns für die Konstituierung unterschiedlicher Gruppen und Kollektive deutlich wird. Zum anderen bildet das mittelalterliche Totengedenken ein aufschlussreiches Anschauungs- und Studienobjekt für die Verzahnung von kommunikativem und kulturellem Gedächtnis: Die listenartigen und kalendarischen Aufzeichnungen der Memorialüberlieferung sind der Ausdruck kommemorativer Praktiken, deren Ausgangspunkt ein allgemein verbreitetes Totengedenken bildet, das jedoch vermittels besonderer Träger-

\footnotetext{
$47 \quad$ Oexle 1983, 35-48.

$48 \quad$ Borgolte 1993, bes. 8-18.

49 Borgolte 2005, 26f. und die Beiträge im Sammelband von Borgolte 2005 (a)

50 Eine die Regel bestätigende Ausnahme bildet der Beitrag von Oexle 1995, der die Rolle des kulturellen Gedächtnisses in den Kulturwissenschaften um 1900 thematisiert und in diesem Zusammenhang auch auf die Arbeiten von Halbwachs und Warburg sowie deren Bedeutung für Assmanns Theorie des kulturellen Gedächtnisses eingeht (vgl. ebd., 22-33). Umgekehrt hat die mediävistische Memoriaforschung nur wenig Aufmerksamkeit in der kulturwissenschaftlichen Erinnerungsforschung gefunden; vgl. die Bemerkungen von Borgolte 1998, 198.
} 
gruppen eine rituelle Verfestigung, Einschreibungen in die kalendarische Zeit und damit Formen annimmt, die seinen Übergang in das kulturelle Gedächtnisses markieren. Wie auch Jan Assmann selbst gesehen hat, ist das Totengedenken daher in besonderer Weise geeignet, um Übergangsformen zwischen dem kommunikativen und dem kulturellen Gedächtnis sichtbar zu machen. ${ }^{51}$

Umgekehrt stellt sich jedoch die Frage, ob eine Konzeptualisierung von Erinnerung als Gabentausch, auf der ihre kategoriale Bedeutung als „,totales Phänomen“ in der mediävistischen Memoriaforschung wesentlich beruht, differenziert genug ist, um kulturspezifische Ausprägungen und Eigenarten des Erinnerns zu erfassen. Virulent wird diese Frage vor allem angesichts der bei den maßgeblichen Vertretern dieser Forschungsrichtung ausgeprägten Tendenz, die Provenienz mittelalterlicher Praktiken und Vorstellungen aus der frühchristlichen und der griechisch-römischen Antike zu betonen und zum Bild einer durch Gabentauschbeziehungen miteinander verbundenen Handlungsgemeinschaft von Lebenden und Toten zu verbinden, die für die Vormoderne insgesamt charakteristisch gewesen und erst in der Sattelzeit des 18. Jh. nachhaltig erschüttert worden sei. ${ }^{52}$ Gerade mit Blick auf die These von der Kulturbedeutung der Memoria wird man jedoch Bedenken haben, Beobachtungen zur mittelalterlichen Geschichte ohne weiteres auf die griechischrömische Antike zu beziehen, und stattdessen stärkeres Gewicht auf die kulturspezifischen Differenzen legen, in denen die Eigenarten zwischen antiker und mittelalterlicher Memorialkultur schärfer hervortreten. Bei näherer Betrachtung erweist sich das Modell des memorialen Gabentauschs als ungeeignet, um darauf die These von der inneren Einheit einer vormodernen Erinnerungskultur zu gründen: Sowohl die Einbeziehung der Toten in einen als gegenwärtig erfahrbaren reziproken Handlungszusammenhang als auch die Semantik des Erinnerns als fürbittendes Gedenken sind Spezifika frühchristlicher und mittelalterlicher Memoria, die sich nicht auf die griechisch-römische Kultur übertragen lassen. ${ }^{53}$ Erinnerung ist danach keine Kategorie, die eine

51 J. Assmann 21997, 61: „Es (scil. das Totengedenken) nimmt offenbar eine Zwischenstellung ein zwischen diesen beiden Formen des sozialen Gedächtnisses. Das Totengedenken ist ,kommunikativ', insofern es eine allgemein menschliche Form darstellt, und es ist ,kulturell ${ }^{`}$ in dem Maße, wie es seine speziellen Träger, Riten und Institutionen ausbildet.“

52 Zur Umbruchszeit des 18. Jh. s. Oexle 1983, 22-25, 71-77; dens. 1985, 98-100; dens. 1995, 54-57; Borgolte 1988, 87-91. Zur Betonung der Kontinuitätslinien zwischen Antike und Mittelalter vgl. noch zuletzt Borgolte 2005 (a), 13.

53 Vgl. dazu u., S. 520-538. In den letzten Jahren ist in den Forschungen zum antiken Christentum im Kontext der kaiserzeitlichen und spätantiken Gesellschaft die Gegenüberstellung „christlich pagan“ als Deutungsschema in den Hintergrund gerückt: Stattdessen wird die Bedeutung von identitätsstiftenden Faktoren wie Sozialstatus, Bildung oder Geschlecht, die quer zu den religiös begründeten Identitätskonstruktionen liegen, stärker hervorgehoben (vgl. zuletzt G. Clark 2004, bes. 8-15). Speziell mit Blick auf den antiken Totenkult ist diese Position zuletzt von Rebillard 2003 nachhaltig vertreten worden. Der Hinweis darauf, dass zahlreiche Lebensbereiche bis in die Spätantike hinein nicht religiös qualifiziert waren bzw. religiöse Qualifikationen nicht wirksam 
weitgespannte kulturelle Kontinuität von der Antike zum Mittelalter begründen könnte, im Gegenteil: Sie ist ein Indikator für einen epochalen Wandlungs- und Transformationsprozess, dessen Stufen im Zuge der spätantiken Entwicklung beschritten wurden.

Aus diesen Überlegungen und Beobachtungen resultieren Thematik, Fragestellung und Ziel der vorliegenden Studie. Ihr Gegenstand ist eine spezifische Form des Totengedenkens, der spätantike Heiligenkult, der in besonderer Weise Aufschlüsse über den Zusammenhang von kollektiver Erinnerung und Identitätsstiftung erlaubt. Aufgrund seiner Popularität und Bedeutung bildete er nicht nur den Kristallisationspunkt einer Vielzahl von Gruppenidentitäten. Als eine besondere Ausprägung des Totenkults brachte der spätantike Heiligenkult auch rituelle Kommemorationsformen und literarische Gattungen hervor, die ihn im kulturellen Gedächtnis verankerten. Seine Ausbildung fällt zudem in eine Zeit, in der Martyrien noch zum gegenwärtigen Erfahrungshorizont der christlichen Gemeinden gehörten, bevor - nach dem Ende der tetrarchischen Verfolgungen zu Beginn des 4. Jh. - der Märtyrer (im wesentlichen) ${ }^{54} \mathrm{zu}$ einem Gegenstand der Vergangenheit wurde. Diese frühe Entwicklungsphase der Heiligenverehrung erlaubt Einblicke in unterschiedliche Stufen kommunikativer und kultureller Erinnerung, an deren Beginn die Märtyrer und Confessoren noch in intensive Kommunikations- und Interaktionsbeziehungen eingebunden waren, aus denen sich in Form von Texten und Riten ,geformte Erinnerungsfiguren eines kulturellen Gedächtnisses entwickelten. Diese Eigenschaften machen den spätantiken Heiligenkult zu einem geeigneten Anschauungsobjekt für Fallstudien, in denen sowohl die Zusammenhänge von Erinnerung und unterschiedlichen Formen kollektiver Identitätsstiftung als auch Übergänge vom kommunikativen in das kulturelle Gedächtnis beleuchtet werden können.

Gleichzeitig bietet der spätantike Heiligenkult aber auch die Gelegenheit, den heuristischen Wert des Konzepts von Erinnerung als kultureller Leitkategorie zu erproben. Er ist das Phänomen einer Transformationszeit, die sich auf zahlreichen Feldern der gesellschaftlichen, politischen, ökonomischen und

wurden, ist ebenso richtig wie die Tatsache, dass die christlichen Gemeinden bereits von ihrer Entstehung an den Entwicklungsbedingungen ihrer jeweiligen Umwelt unterlagen. Dennoch erscheint unstrittig, dass manche Phänomene nicht erklärt werden können, ohne jüdisch-christliche Traditionen als eigenständigen kulturellen Faktor zu berücksichtigen - für den Aspekt von Erinnerung und Gedenken gilt dies ganz zweifellos.

54 Infolge der innerkirchlichen Auseinandersetzungen nach der, konstantinischen Wende‘ blieb die Figur des Märtyrers oder Confessors auch noch in der Folgezeit präsent, um von den jeweils unterlegenen Seiten für ihre Opfer in Anspruch genommen zu werden. Dies gilt z. B. für die Donatisten in Nordafrika (vgl. Tilley 1996) oder für einige italische Bischöfe wie Eusebius v. Vercellae und Dionysius v. Mailand, die Position gegen die homöische Politik Constantius' II. bezogen und 355 verbannt wurden (s. Picard 1988, 683f.). Dennoch war die eigentliche Zeit der Märtyrer mit dem Jahr 313 vorbei (vgl. Markus 1990, 24, 90-95). 
kulturellen Entwicklung bemerkbar machte. ${ }^{55}$ Auch wenn die Spätantike dabei durchaus den Charakter einer eigenen Epoche hat und nicht einfach als Übergangszeit hin zur frühmittelalterlichen Geschichte betrachtet werden sollte, und trotz der Berücksichtigung von Unterschieden, die nicht nur zwischen der westlichen und östlichen Mittelmeerwelt bestanden, sondern auch auf regionaler Ebene wirksam wurden, dürfte unbestreitbar sein, dass sich in der Spätantike allgemeine Entwicklungen vollzogen, die einen grundlegenden kulturellen Wandel indizieren und als schrittweiser Auflösungsprozess der antiken Kultur zu verstehen sind. Als ebenso unbestreitbar kann gelten, dass christliche Traditionen, so sehr sie auch ihrerseits durch die antike Kultur vermittelt und bedingt waren, daran einen maßgeblichen Anteil hatten. Im folgenden soll die These zur Disposition gestellt werden, dass Erinnerung eine zentrale Kategorie dieses kulturellen Wandels ist. Um dies zu untermauern, wird der Heiligenkult abschließend idealtypisch auf diejenige kulturelle Formation bezogen, die als ein wesentliches Charakteristikum für die Kultur der antiken Mittelmeerwelt insgesamt gelten kann und die bereits seit dem frühen 19. Jh. Anlass zu Kontinuitätsdebatten und der Frage nach dem epochalen Fortbestehen oder Wandel zwischen Spätantike und Frühmittelalter bietet: die Stadt. ${ }^{56}$ Neben die traditionelle Konzentration auf Aspekte der urbanistischen Entwicklung, der politisch-administrativen Funktion und der ökonomischen Bedeutung von Städten im Übergang von der Spätantike zum Frühmittelalter tritt damit ein weiteres Kriterium, das die in der althistorischen, archäologischen und mediävistischen Forschung intensiv diskutierte Frage: „Was ist die antike Stadt?“57 von einer anderen Richtung her angeht: Wie veränderte sich die Memorialkultur, die das städtische Leben und Er-

55 Für einen konzeptionellen Versuch, die verschiedenen Felder mit ihren unterschiedlichen Entwicklungsgeschwindigkeiten zueinander in Beziehung zu setzen, und für das Plädoyer, trotz der Komplexität der damit verbundenen Probleme an der Kategorie der „Epoche“ bzw. des „Epochenwandels“ festzuhalten, vgl. C. Meier 1973. Eine ausgezeichnete und umfassende, nicht allein auf die literaturwissenschaftliche Perspektive beschränkte Diskussion des Epochencharakters der Spätantike bietet Herzog 1989.

56 Vgl. die ebenso knappe wie treffende Bemerkung von Averil Cameron 1993, 152: „Unlike the medieval world, the civilisation and high culture of classical antiquity, and thus also of the Roman empire, rested on a network of cities. The end of classical antiquity thus seems to imply the end of classical cities, and vice versa. “ Die Anfänge einer wissenschaftlichen Auseinandersetzung um Wandel oder Fortbestehen der Stadt im Übergang zum Frühmittelalter gehen bis auf die Kontroversen zur Stadtverfassung in der ersten Hälfte des 19. Jh. zurück und sind vor allem mit den Namen Friedrich Karl von Savigny und Karl von Hegel verbunden (vgl. Nippel 1991, 26).

57 Für eine Problematisierung der Frage und eine Forschungsbilanz aus althistorischer Perspektive

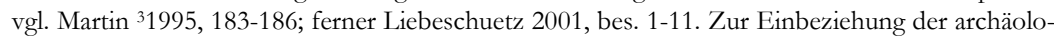
gischen Aspekte (Urbanistik, Stadtarchitektur) städtischer Entwicklung in der Spätantike vgl. zuletzt Haug 2003, bes. 11-41. Zum aktuellen Stand der Kontinuitätsdebatte aus mediävistischer Sicht vgl. Wickham 2005, 591-692. 
scheinungsbild der griechischen und römischen Stadt in elementarer Weise prägte und sie zu einem Erinnerungsraum sui generis machte?

\section{1. 2. Erinnerungsräume}

Wenn eingangs mit Blick auf das Konzept der Erinnerung festgestellt wurde, dass es in den letzten Jahren konjunkturelle Höhenflüge erlebt hat, so gilt dies vom „Erinnerungsort“ bzw. „-raum“ in potenzierter Form. Ihre populärste Ausprägung hat die Begrifflichkeit zweifelsohne in Pierre Noras Projekt der „lieux de mémoire“ gefunden, das in den letzten Jahren in Deutschland und zahlreichen anderen Ländern zu vergleichbaren Konzeptionen nationaler „Erinnerungsorte“ geführt hat. ${ }^{58}$ Die Begriffsprägung geht darauf zurück, dass ein Ort über seine Eigenschaft als eine topographisch lokalisierbare Begebenheit hinaus auch eine gedächtnisstrukturierende Bedeutung hat. Bereits in der antik-humanistischen Tradition der Mnemotechnik begegnet die Vorstellung, dass das Gedächtnis in Räume unterteilt sei und bestimmte Informationen dieses Gedächtnisspeichers durch das Begehen der Gedächtnisorte, an denen sie abgelegt wurden, abgerufen werden könnten. ${ }^{59}$ Verortung, verstanden als eine Einlagerung und Speicherung im Raum des Gedächtnisses, wird also - ähnlich wie in anderen Fällen von sprachlicher Raummetaphorik $^{60}$ - in einem übertragenen Sinn gebraucht, der sich von der Vorstellung einer physisch-topographischen Lokalisierung löst.

Gedächtnisorte und topographischer Raum schließen einander zwar nicht aus: Bereits Cicero verband seine Reflexion über die vergegenwärtigende Kraft von Orten, die bestimmte Personen an ihren historischen Wirkungsstätten in Erinnerung riefen, mit der Bedeutung von Orten in der Mnemotechnik. ${ }^{61}$ Dennoch ist für die Rede von Erinnerungsorten charakteristisch, dass sie keiner topographischen Definition folgt, sondern den Raumbegriff aus der Mnemotechnik bezieht und in unterschiedlicher Weise metaphorisch

$58 \quad$ Für das von Nora abweichende Konzept der deutschen Erinnerungsorte vgl. u., Anm. 62. Für vergleichbare Projekte in Italien, Dänemark, den Niederlanden und Österreich s. den Hinweis von François 2005, 12.

59 Zum mnemotechnischen Verfahren und seiner Bedeutung bis ins 18. Jh. vgl. A. Assmann 1999, 27-29.

60 Exemplarisch etwa in der oben skizzierten Rede Bourdieus vom „sozialen Raum“; zur Konzeptualisierung der Kategorie „Raum“ in ihren unterschiedlichen Ausprägungen vgl. zuletzt Damir-Geilsdorf/Hendrich 2005, 26-32.

${ }_{61}$ Vgl. Cic., fin. 5, 1f. anlässlich einer Begehung der Akademie in Athen: tanta vis admonitionis inest in locis, ut non sine causa ex iis memoriae ducta sit disciplina. Die Verbindung des Gedächtnisses der Orte mit der Mnemotechnik bleibt allerdings assoziativ; Cicero geht im Kontext der Stelle nicht weiter auf die Gedächtniskunst ein. 
ausdeutet. ${ }^{62}$ Nora versteht daher unter den „lieux de mémoire“ neben topographisch lokalisierbaren Orten, Gedenkstätten und Denkmälern auch historische Persönlichkeiten, symbolische Manifestationen der französischen Geschichte (z.B. die Tricolore, die Marseillaise) ebenso wie die Medien ihrer Vermittlung (Geschichtswerke, Feiertage) und nationale Frankreichbilder. In ähnlicher Weise hat auch das Projekt der deutschen Erinnerungsorte historische Stätten, Ereignisse, Persönlichkeiten, Kunstwerke und Leitbegriffe zum Gegenstand, die in lockerer Verbindung um einzelne Themenkreise herum gruppiert sind.

Wenn im folgenden ebenfalls von „Erinnerungsräumen“ die Rede ist, dann nicht, um in vergleichbarer Weise die metaphorische Valenz des Ortsund Raumbegriffs für die Struktur eines kollektiven Gedächtnisses auszuloten. Der Sprachgebrauch knüpft vielmehr an die Bedeutung von physisch und topographisch lokalisierbaren Orten für Prozesse der Erinnerung und kollektiven Identitätsstiftung an, auf die bereits Maurice Halbwachs hingewiesen hat. Dass Orte und materielle Gegenstände als bevorzugte Kristallisationspunkte von Erinnerung und der sich im Erinnern vollziehenden Identitätsstiftung fungieren, hat Halbwachs einseitig mit ihrer Resistenz gegen Veränderung, die sie verkörpern (bzw. suggerieren), begründet: Wie das Gedächtnis selbst betonen Orte und Dinge Kohärenz und Kontinuität im Wandel der Zeiten und liefern auf diese Weise gleichsam räumliche Abbilder derjenigen Integrationsleistungen, die für das Konzept personaler wie kollektiver Identität konstitutiv sind. ${ }^{63}$ Dies ist zwar dahingehend zu präzisieren, dass Orte nicht nur der affirmativen Identitätsstiftung dienen, sondern auch die gegenteilige Erfahrung vermitteln können. Dies gilt für reale Orte wie Ruinen und Überreste, die Diskontinuitäten versinnbildlichen, ebenso wie für literarische Ortsbegehungen, die in ähnlicher Weise mit der Differenz zwischen imaginierter Vergangenheit und erfahrbarer Gegenwart operieren. Bezeichnend ist jedoch, dass auch in dieser Variante der Ort ein bevorzugtes Mittel ist, um dem Thema „Identität“ symbolischen Ausdruck zu verleihen. Ob zur Affir-

${ }^{62}$ Vgl. die unterschiedlichen Akzentsetzungen von Nora 1990 und François/Schulze 2001. Nora versteht seine „lieux de mémoire“ als Orte, ,an denen sich das Gedächtnis lagert oder in das es sich zurückzieht“; diese Orte ergeben zusammengenommen einen Erinnerungsraum (Nora 1990, 11, 16). François/Schulze 2001, 18 hingegen knüpfen an den Begriff „Erinnerungsort“ die Vorstellung, dass er keine ,abgeschlossene Realität“ darstelle, sondern erst durch die Bezüge in einem größeren Raum Bedeutung und Sinn erhalte. Sowohl Nora als auch François/Schulze beziehen den Begriff aus der antik-humanistischen Mnemotechnik (Nora 1990, 7; François/Schulze 2001, 17).

63 Halbwachs 1967 (1950), 127-163. Zum Gedächtnis, das im Unterschied zur Geschichte Differenzen und Wandel ausblendet, vgl. o., S. 6f..; zur Definition von Identität als gelungener Integrationsleistung vor dem Hintergrund von synchroner und diachroner Differenzerfahrung s. o., Anm. 32. 
mation oder zur Infragestellung von Identität, Orten kommt in beiden Fällen eine identitätsrelevante Bedeutung zu.

Diese allgemeine Beobachtung, dass topographisch bestimmbare Orte und materiell erfahrbare Gegenstände und Monumente identitätsrelevante Erinnerungsprozesse in Gang setzen, lässt sich weiter ausdifferenzieren, um den Charakter dieser Orte und im Raum lokalisierbarer Objekte genauer zu bestimmen. Dabei wird in der Forschung meist unterschieden zwischen Denkmälern, die mit der Intention errichtet wurden, die Erinnerung an ein Ereignis lebendig zu halten, und Orten, die zwar Anlagerungspunkte von Erinnerung bilden, jedoch nicht eigens zum Zweck der Erinnerung ins Leben gerufen worden sind, und die in unterschiedlicher Weise als „Landschaft“" oder auch als „Erinnerungsorte“ aufgefasst werden. ${ }^{64}$

Die räumlich vermittelte Heiligenerinnerung im spätantiken Rom ist in beiden Ausprägungen fassbar. Sie fand auf der einen Seite Ausdruck in Monumentalisierungen, die von den außerhalb der Stadt gelegenen Gräbern der Märtyrer als räumlichem Fokus ihren Ausgang nahmen und von dort, vermittelt durch Reliquientranslationen, in die Stadt „einwanderten“, um im städtischen Raum in Form von Oratorien und Kirchen neue Zentren der Verehrung zu bilden. Auf der anderen Seite entstand seit dem 5. Jh. eine umfangreiche Produktion an römischen Heiligenviten, die in stereotyper Form das Lebensschicksal ihrer Protagonisten mit bestimmten Schauplätzen verknüpften und auf diese Weise ein dichtes Bild von Erinnerungsorten entwarfen. ${ }^{65}$ Dennoch stellt sich die Frage, ob mit dieser Differenzierung zwischen Monumenten und Erinnerungsorten das heuristische Potential des Erinnerungsraums bereits ausgeschöpft ist, oder ob die spätantike Heiligenmemoria nicht noch in einer anderen und grundlegenderen Form als Indikator für den Wandel des städtischen Raums dienen kann. Es wird daher im folgenden vorgeschlagen, die antike Stadt in einem umfassenderen Sinne als Erinnerungsraum zu verstehen, der durch die Konzepte des Erinnerungsorts und des Denkmals, die beide auf einem retrospektiven Erinnerungsbegriff beruhen, nicht hinreichend erfasst wird.

So ist für jüngere Forschungen zum Erinnerungsraum des republikanischen Rom kennzeichnend, dass sie dem Gedächtnis einen intentionalen Vergangenheitsbezug zugrunde legen und den Begriff des Denkmals eng an diese Intentionalität koppeln, um auf diese Weise innerhalb des monumentalen Er-

64 Alcock 2001, 326f., dies. 2002, 28-32 trennt zwischen „Monumenten“ und „Landschaften“, Walter 2004, 131-137; 155-179 zwischen „Denkmälern“ und „Erinnerungsorten“. Während Alcock unter dem Begriff der „Landschaft“ die städtisch, bäuerlich oder sakral geprägte Siedlungsstruktur versteht, auf die sich einzelne Monumente verteilen, ist Walters „Erinnerungsort“ auf bestimmte topographische Lokalitäten bezogen; in diesem Sinne wird der Begriff auch im folgenden verwendet.

$65 \mathrm{Zu}$ den gesta martyrun Romanorum und ihren Entwürfen einer römischen Erinnerungslandschaft vgl. u., Kapitel VI. 2. 
scheinungsbildes Denkmäler von Überresten abzugrenzen, die zwar eine diffuse Vorstellung von Alter und Ehrwürdigkeit vermittelten, jedoch nicht explizit auf historische Ereignisse verwiesen. ${ }^{66}$ Aus dieser Perspektive besteht der Erkenntniswert von Monumenten für die Erforschung einer Erinnerungskultur darin, dass sie memoriale Potentiale verkörpern, die ein retrospektives Erinnern ermöglichen, indem sie entweder - retrospektiv - in rückblickender Erinnerung an eine Person bzw. ein Ereignis errichtet wurden oder aber - prospektiv - zu dem Zweck, ein zeitgenössisches erinnerungswürdiges Ereignis im Gedächtnis zu erhalten. ${ }^{67}$

Es ist jedoch fraglich, ob diese konzeptionelle Eingrenzung des Gedenkens auf retrospektive Erinnerungsleistungen und intentionale Vergangenheitsbezüge das in der städtischen Monumentallandschaft verkörperte Erinnern nicht zu eng fasst, da sie weitgehend von den Mechanismen der antiken Memorialkultur selbst absieht, die zur Entstehung von Denkmälern und Monumenten maßgeblichen Anlass gab. Denkmäler und Monumente in antiken Städten waren eingebunden in einen dynamischen und umfassenden Prozess memorialer Praktiken, für den die Forschung den modernen Kunstbegriff des „Euergetismus“ geprägt hat. Die mit diesem Ausdruck angesprochene hohe Bereitschaft städtischer Eliten, Leistungen im Interesse ihrer Stadt und ihrer Mitbürger zu erbringen, prägte das kulturelle Leben und urbane Erscheinungsbild in nachhaltiger Art und Weise und machte den städtischen Raum zu einem Erinnerungsraum in einem nicht nur geschichtskulturellen Sinne. In zahlreichen Bauten, Statuen und vor allem Inschriften ${ }^{68}$ materialisierten sich nicht nur Leistungsbereitschaft und Stifterwillen, sondern vor allem auch die Erinnerung, die man aus Dank für die erbrachten Leistungen schuldete. Diese intensive Produktion von Memoria erstreckte sich nicht nur auf den öffentlichen Raum, sondern auch auf private Wohnbereiche, die religiöse Topographie und die Grabkultur. Ein seit der Kaiserzeit blühendes Vereinswesen ermöglichte es auch begüterten Personen unterhalb der städtischen Führungsschicht, im Wirkungskreis ihres Kollegiums als Wohltäter aufzutreten. Diese Verbindung von urbaner Landschaft und Gedenken, das sich - in

${ }_{66}$ Vgl. T. Hölscher 2001, bes. 184f.: „Im genauen und wörtlichen Sinn bedeutet das ,Denk-Mal‘, das ,Monument', den expliziten und intentionalen Verweis auf eine als solche verstandene Vergangenheit“; zustimmend Walter 2004, 131, der Intentionalität zum Kriterium auch für den Erinnerungsort erhebt (ebd. 155).

67 Dass auch in diesem Fall prospektiver Memoria das retrospektive Erinnern im Zentrum des Forschungsinteresses steht, macht Walter 2004, 133 deutlich: „Für die Geschichtskultur interessant sind die Differenzen, die sich etwa aus dem Gegenstand des Denkmals und seiner anfänglichen Aussageabsicht, dem zeitlichen Abstand zwischen dem Ereignis und seiner, Verewigung' oder der verstrichenen Zeit zwischen der Entstehung des Monuments und seiner Wahrnehmung durch den Betrachter ergeben."

68 Zur bedeutenden Rolle von Inschriften im Kontext der antiken Stadtkultur vgl. Eck 1997 (a), 99; zu ihrer memorialen Funktion als monumenta vgl. Cooley 2000, 7. 
Anlehnung an Oexle - nicht als geschichtskulturelles Erinnern, sondern als eine soziale Gabe verstehen lässt, erfasste damit weite Bereiche und Bevölkerungsschichten der Stadt.

Mit Blick auf die Monumentallandschaft des spätantiken Rom haben in jüngerer Zeit vor allem Aspekte des intentionalen bzw. geschichtskulturellen Umgangs mit der Vergangenheit Aufmerksamkeit gefunden. Unter dem Stichwort der „Musealisierung“ wurden sowohl konservierende Baumaßnahmen als auch Wahrnehmungen der ,antiken“ Monumente Roms auf die Semantik von Vergangenheitsbezügen hin in den Blick genommen. ${ }^{69}$ Die vorliegende Studie zur Heiligenerinnerung im spätantiken Rom verfolgt in Teilen einen ähnlichen Ansatz, wenn sie nach der Bedeutung der Heiligen und ihrer Verehrungsstätten für die Identität der Stadt und der Gemeinde Roms fragt. ${ }^{70}$ Sie legt jedoch zugleich einen weiteren Begriff von Erinnerung zugrunde, indem sie der Frage nachgeht, inwieweit die Heiligenerinnerung im Kontext der - nicht auf das intentionale Erinnern beschränkten - Memorialkultur der antiken Stadt neue Akzente setzte. ${ }^{71}$ Die hierin zutage tretenden Differenzen bilden Ausgangspunkt für die grundlegende Frage nach einer Integrierbarkeit des Heiligengedenkens und christlicher Konzeptualisierungen von Erinnerung in die antike Erinnerungskultur. ${ }^{72}$ Dabei wird deutlich werden, dass die Krise der spätantiken Stadt zu einem nicht unerheblichen Teil das Ergebnis einer veränderten Auffassung von der Memoria als sozialer Gabe war - ein Strukturwandel, der nicht ohne Auswirkungen auf den Erinnerungsraum der antiken Stadtkultur blieb.

\section{2. Inhaltliche Vorbemerkungen}

Nach diesen grundsätzlichen Ausführungen zur Zielsetzung und Methode sollen im folgenden die inhaltlichen Aspekte der in dieser Studie verfolgten Thematik zur Sprache kommen. Dabei wird zunächst kurz rekapituliert, warum der spätantike Heiligenkult als ein besonders aussagekräftiges historisches Phänomen gelten kann, um Prozesse kollektiver Identitätsstiftung durch Erinnerung zu analysieren und die Frage nach der kulturgeschichtlichen Dimension von Erinnerung zu thematisieren. Die daran geknüpften Bemer-

69 Zur musealisierenden Konservierung von Teilen des Forum Romanum vgl. Bauer 1996, bes. 110, 141. Die angekündigte Studie von Ralf Behrwald, Die Stadt als Museum. Die Wahrnehmung der Monumente Roms in der Spätantike, Berlin 2007 verspricht eine systematische Aufarbeitung der Thematik. Beiläufigere Bemerkungen zur Musealisierung Roms finden sich auch bei Février 1993, 51; Witschel 2001, 122.

70 So besonders in den Kapiteln III und IV.

71 Vgl. dazu vor allem die Kapitel II, V und VI.

72 Kapitel VII. 
kungen dienen dem Zweck, die spätantike Heiligenmemoria insgesamt als historisches Phänomen im Kontext der im vorigen diskutierten Aspekte und Probleme der kulturwissenschaftlichen Erinnerungsforschung zu verorten, und bilden damit den Ausgangspunkt für die daran anschließenden Ausführungen zum konkreten Untersuchungsgegenstand, dem Heiligenkult im Rom des 3. bis 5. Jh. n. Chr. Diese inhaltlichen Ausführungen wiederum verfolgen ein doppeltes Ziel: Zum einen konzentrieren sie sich darauf, eine Orientierung über die in den einzelnen Kapiteln behandelten thematischen Schwerpunkte zu vermitteln und ihre Auswahl zu begründen. Zum anderen soll deutlich werden, dass die hier verfolgte Fragestellung eine zwischen Strukturund Entwicklungsgeschichte angesiedelte Form der Darstellung erfordert und damit ein bestimmtes ,Format' vorgibt, das beide Aspekte in adäquater Weise $\mathrm{zu}$ berücksichtigen sucht, ohne dass sie jedoch vollständig in eine geschlossene Geschichte der Heiligenerinnerung im spätantiken Rom integrierbar wären.

\section{2. 1. Der spätantike Heiligenkult als Gegenstand der Erinnerungsforschung}

Die folgenden Bemerkungen wollen keinen historischen Abriss zur Entwicklung des Heiligenkults in der hohen Kaiserzeit und der Spätantike bieten. ${ }^{73}$ Sie beschränken sich bewusst auf eine sehr knappe Skizze, die von regionalen und diachronen Differenzierungen absieht und sich darauf konzentriert, diejenigen Strukturelemente hervorzuheben, die für die im Begriff des „Erinnerungsraums" angesprochene Verbindung von Erinnerung, Identität und räumlichen Repräsentationen konstitutiv sind.

Erinnerungsbezug: Die Kategorie der Erinnerung erfährt im Heiligenkult eine scharf umrissene Ausprägung, durch die der wenig spezifische Begriff des kollektiven Gedächtnisses präzisiert, von Bereichen eines kollektiv geteilten Wissens abgegrenzt und auf ein bestimmtes historisches Phänomen bezogen werden kann. Denn Heiligenkult bedeutet per definitionem nicht nur Verehrung des Heiligen, sondern vor allem Gedenken und Erinnerung an ihn: Sein Kult entwickelte sich - nicht nur in Rom - als ein Sonderfall der christlichen Totenkommemoration ${ }^{74}$ und war damit in einem gesellschaftlichen Hand-

73 Ausgezeichnete Überblicke bieten Baumeister 1988; van Uytfanghe 1996.

74 Der Begriff des christlichen Heiligen erfuhr in der antiken Mittelmeerwelt regional- und kulturspezifisch unterschiedliche Ausprägungen (vgl. dazu die Bemerkungen von Kretschmar 1977, 88-92). Im Westen des römischen Reichs entwickelte sich seit der Wende vom 2. zum 3. Jh. der Märtyrer zum Inbegriff des Heiligenideals (vgl. Saxer 1984): Sein Kult konzentrierte sich hier auf den Todestag, an dem man des Märtyrers und seines Martyriums gedachte. In Rom setzte die Märtyrerverehrung zu Beginn des 3. Jh. ein, zur selben Zeit, in der sich auch ein kalendarisches Bischofsgedenken entwickelte. Damit ist sicher, dass auch in Rom der Heiligenkult die 
lungsbereich angesiedelt, der elementar von Semantiken und Praktiken des Erinnerns geprägt war. Anders als beim Gedenken an „normale“ Verstorbene, das gewöhnlich einem periodischen Vergessen anheim fiel, beschränkte sich das Gedenken an die Heiligen zudem nicht auf die Phasen und Erscheinungsformen eines kommunikativen Generationengedächtnisses: Die Verehrung des Märtyrers vollzog sich in der Form eines regelmäßig wiederkehrenden Gedenkens, als eine kalendarische Kommemoration seines Todestages. ${ }^{75}$ Auf diese Weise wurde der Heilige der zeitlichen Struktur und dem Gedächtnis von christlichen Gruppen eingeschrieben, die seiner in regelmäßigen Abständen gedachten, und damit Teil des kulturellen Gedächtnisses. Dasselbe gilt für die hagiographischen Texte, die ursprünglich in enger Verbindung mit dem rituellen Gedenken an einen Heiligen standen, indem sie zum Jahrestag seiner Passion gelesen wurden, sich jedoch bald zu einer davon unabhängigen Literatur verselbständigten und damit ihrerseits zu einem eigenständigen Medium des Heiligengedenkens im kulturellen Gedächtnis wurden. ${ }^{76}$ Das antike Heiligengedenken stand somit im Zentrum unterschiedlicher Praktiken und Medien des Erinnerns, die im Bereich sowohl des kommunikativen als auch des kulturellen Gedächtnisses anzusiedeln sind und deren kulturelle Ausprägungen alle wesentlichen Erscheinungsformen des kulturellen Gedächtnisses - Ritus, Text und Monument ${ }^{77}$ (s. u. Raumbezug) - umfassen.

Form eines Totengedenkens annahm. Die Frage nach der historischen Relation von Bischofsund Märtyrergedenken in Rom kann noch nicht als geklärt angesehen werden. Stuiber 1960 plädiert dafür, dass das im frühen 3. Jh. einsetzende Bischofsgedenken den Anstoß zur Märtyrerkommemoration gegeben habe; anderer Ansicht ist Février 1991, 64f., der umgekehrt meint, das Bischofsgedenken habe sich in Rom aus dem Märtyrergedenken heraus entwickelt. Zur Entwicklung des Märtyrerkults aus dem Totenkult vgl. Kirsch 1900, 91f.; Dölger 1922, 568; Klauser 1927, 140; Delehaye 1933, 33f.; anders zuletzt Karpinski 1987, 80-94 mit wenig überzeugenden Argumenten.

75 Für die Entwicklung der antiken Heiligenkalender ist immer noch grundlegend Achelis 1900; s. zuletzt Auf der Maur 1994, 102f. (Literatur). Aus Rom ist eines der frühesten Beispiele mit den beiden Depositionslisten (depositio martyrum und depositio episcoporum) aus dem Chronographen des Jahres 354 erhalten (MGH AA IX, 70-72).

76 Die enge Verbindung von Text und Ritual, durch die dem Text seinerseits eine rituelle Bedeutung zuwächst, wird besonders deutlich im Prolog der passio Perpetuae: Die Lektüre der Passio soll bei denen, die das Martyrium der Heiligen selbst erlebt haben, die Geschehnisse in Erinnerung rufen; für diejenigen, die zum erstenmal vom Leiden der Heiligen erfahren, sind es das Gehörte und damit der Text selbst, die eine communio mit den Heiligen bewirken (vgl. Pass. Perp. 1, 6: quod audivimus et contrectavimus, annuntiamus et vobis, fratres et filioli, uti et vos, qui interfuistis rememoremini gloriae Domini, et qui nunc cognoscitis per auditum communionem habeatis cum sanctis martyribus). - Zu den informativsten Darstellungen der unterschiedlichen Literaturgattungen, die die antike Hagiographie hervorbrachte, zählt immer noch Delehaye 21966. Zur spätantiken Hagiographie in Rom s. u., S. 258-269, 432-447.

77 S. o., S. 7 f. 
Gruppenbezug: Die Figur des Märtyrers entwickelte sich seit dem 2. Jh. zu einem der wichtigsten identitätsstiftenden Symbole der Kirche und der einzelnen Gemeinden, in denen die ecclesia Gestalt gewann. ${ }^{78}$ Zum semen christianorum $^{79}$ wurden die Blutzeugen deswegen, weil sie das der Identitätsstiftung inhärente Moment der Abgrenzung ${ }^{80}$ in besonderer Weise verkörperten: Durch die Thematisierung von Gewalt - eines Phänomens, das jenseits aller kulturanthropologischen Differenzierungen als eine der radikalsten Ausdrucksformen von Abgrenzung einzustufen ist - gewann auch die Identität des Christlichen in der Person des Märtyrers eine scharfe Ausprägung. Diese identitätsstiftende Funktion tritt auch in der Erinnerung an die Märtyrer und den Memorialpraktiken des Heiligenkults hervor. Besonders deutlich wird dies in der literarischen Verarbeitung der Martyrien. Schon mit dem Einsetzen der ersten Märtyrerakten, also seit dem frühen 2. Jh., entwickelte sich die Figur des Märtyrers zum Kristallisationspunkt von Identitätsdiskursen, die in geradezu leitmotivischer Wiederkehr auf unterschiedlichen Ebenen dieser Berichte geführt werden: Das stereotype Identitätsbekenntnis des christianus sum, das der Märtyrer vor dem verhörenden Richter abgibt, ${ }^{81}$ die Inversion nichtchristlicher Orte der Identitätsstiftung, die in den Akten zum Schauplätzen des christlichen Bekenntnisses werden, ${ }^{82}$ und der in den Akten immer wieder betonte Gemeinschaftsbezug der Heiligen ${ }^{83}$ sind nur einige der Aspekte, bei denen die Thematisierung von Identität in den hagiographischen Texten selbst wirksam wird. Die Heiligen- und Totenerinnerung involvierte neben den Texten aber auch das Handeln von Gruppen. Ihre bereits früh hervortretende Fähigkeit zur Interzession machte die Heiligen nicht nur zu Adressaten individueller Annäherungen um Fürbitte und Hilfe, sondern blieb zumindest bis zum Ende des hier untersuchten Zeitraums - auf Gruppen und Gemeinschaften bezogen, in die die Heiligen eingebunden waren. Durch die kommemorativen Praktiken, vermittels derer unterschiedliche Kollektive ihre

78 Nock 1933, 192f., misst den Märtyrern für die christlichen Kultgemeinschaften dieselbe identitätsstiftende Bedeutung zu, wie sie Tempel und Priester in den paganen Kulten hatten; zur Relevanz der Märtyrer für die Identität der Kirche vgl. allgemein auch Lane Fox 1986, 441; Hopkins 1998, $196 \mathrm{f}$.

79 Tert., apol. 50, 13.

80 Zum komplementären Verhältnis und den Wechselwirkungen in der Konstruktion von Identität und Alterität vgl. A. Assmann/Friese 1998, 15-21.

81 Vgl. hierzu treffend Shaw 1996, 301: „Every trial was therefore a matter of fixing identities“.

82 Diefenbach 2000, bes. 112-131.

83 Er äußert sich u. a. im Gebet der Heiligen für die Kirche (martyrium Polycarpi 8, 1; passio Fructuosi 3; vgl. Baus 1953, 26f.), in der Vorbildhaftigkeit des Märtyrers für die Mitbrüder (Pass. Mar. Iac. 1), in der von Märtyrerbischöfen vorgenommenen Akzentuierung des Opfergedankens für die Gemeinschaft (passio Irenaei 5, 4f.; die dort angesprochene Dimension des Opfergedankens ist auch für passio Felicis Thibiucensis 37 vorauszusetzen) oder in der - auch in den Märtyrerakten selbst thematisierten - Zusammenkunft der Gemeinde im Gedenken an ihre Märtyrer (vgl. martyrium Polycarpi 18; Pass. Perp. 1f.); für weitere Belege vgl. Pellegrino 1961, 157-162. 
Beziehung zu den verehrten Heiligen zu bestimmen suchten, und durch die Diskurse, in denen die Heiligen als Symbole dieser Gemeinschaften erscheinen, wurden sie zu Bezugspunkten unterschiedlicher kollektiver Identitäten, die von Grabgemeinschaften, die den kaiserzeitlichen Kollegien ähnelten, über einzelne Haushalte und die christliche(n) Gemeinde(n) Roms bis hin zur städtischen Gemeinde Roms reichten. ${ }^{84}$

Raumbezug: Insgesamt ist es für antike Gesellschaften der griechisch-römischen Mittelmeerkultur kennzeichnend, dass Gruppenzugehörigkeiten (beispielsweise als Bürger einer Stadt, als Mitglied eines Kollegiums, als Angehöriger einer Klientel oder als Anhänger einer christlichen Gemeinde) elementar durch Orte vermittelt wurden, die Schauplätze für die Interaktion von Gruppen bildeten und für sie oder auf ihre Initiative hin errichtet wurden (wie etwa städtische Monumente, scholae, domus oder Kirchengebäude). Für den Heiligenkult gilt diese identitätsstiftende Funktion von Orten und Räumen jedoch in besonderer Weise. Seine Eigenschaft als Totenkult brachte von vornherein eine räumliche Ausrichtung mit sich: Das Grab des Märtyrers bildete den Fokus des Gedenkens an ihn und koppelte damit Erinnerung und Identität an einen lokalisierbaren Raum, ${ }^{85}$ der in den Nekropolen außerhalb des antiken Stadtgebietes lag und damit in ein Spannungsverhältnis zum städtischen Raum und den durch ihn vermittelten Identitätskonstruktionen (der Gemeinschaft der Lebenden, der cives Romani, der christlichen Gemeinde der Stadt Rom) treten konnte. Die Verehrung der Heiligen blieb jedoch nicht auf ihre Gräber beschränkt. Mit der Überführung ihrer sterblichen Überreste in städtische Kirchen und Oratorien entstanden neue Pole der Verehrung, die die traditionelle Trennung zwischen dem städtischen Raum und der Nekropolenlandschaft außerhalb der Städte aufbrachen und Orte der Heiligenmemoria zu Bezugspunkten innerhalb der Städte und der Kirchenräume machte. Die dadurch entstehende Erinnerungstopographie spiegelte sich nicht nur in den Monumenten, sondern daneben auch auf der Ebene von Texten wider. In den spätantiken Passionen der römischen Heiligen wurden die Stadt und der städtische Raum zu einer Bühne, auf der die Verfasser ihre Protagonisten agieren (und natürlich sterben) ließen. Dieser legendarische Diskurs lässt deutliche Eigengesetzlichkeiten in der Konstruktion eines literarischen Erinnerungsraums erkennen, der eine eigene Bedeutungsebene neben dem monumentalen und materiellen Erscheinungsbild konstituierte, jedoch nicht vollkommen losgelöst von ihm existierte: In den Legenden werden vielmehr Wahrnehmungen des städtischen Raums fassbar, die mit dem monumentalen

\footnotetext{
84 Hinzu kommen Aspekte personaler Identitätsstiftung durch heilige Patrone, die im Rahmen dieser Studie jedoch nicht verfolgt werden (vgl. Brown 1981, 50-68 u. ö.).

85 Zum Grab und dem Todestag als den beiden fundamentalen „Koordinaten“ der Heiligenverehrung vgl. Delehaye 1934, 7-17.
} 
Erscheinungsbild der Stadt im Übergang zum Frühmittelalter korrespondieren und den damit verbundenen Transformationsprozess auch auf der Ebene der Imagination und Wahrnehmung dokumentieren.

\section{2. 2. Römische Erinnerungsräume im 3. bis 5. Jh. n. Chr.}

Das Zusammenspiel von Raum, Identitätsstiftung und Heiligenerinnerung lässt sich nicht in Form einer einheitlichen und kontinuierlichen Geschichte der Heiligenmemoria im spätantiken Rom schreiben. Dass das Thema der Memoria sich einer seit der Aufklärung gängigen Konzeption von „der“ Geschichte im Sinne eines Kollektivsingulars entzieht, hat sowohl methodische als auch quellenbedingte Gründe. In methodischer Hinsicht sind memoriale Praktiken, Inszenierungen und Medien in ihren jeweiligen Bezügen auf einzelne Kollektive hin zu analysieren, für die sie eine identitätsstiftende Bedeutung erlangt haben. Diese unterschiedlichen Felder kollektiver Identitätsstiftung wiederum sind jedoch in einem $\mathrm{zu}$ hohen Maße durch Eigenentwicklungen gekennzeichnet, als dass man sie ohne weiteres zu einer - durch einheitlichen Verlauf und Richtungssinn charakterisierten ${ }^{86}$ - „Geschichte der Memoria“" verbinden könnte. Umgekehrt jedoch lassen sich diese einzelnen Felder auch nicht in Form eines streng parallel angelegten diachronen Durchlaufs darstellen. Dem steht zum einen das quellenbedingte Problem, dass nicht alle der im folgenden untersuchten Kollektive über den gesamten hier untersuchten Zeitraum hinweg gleichmäßig gut bezeugt sind, im Wege, zum anderen aber auch, dass auf diese Weise das Gewicht und die Wertigkeit von Phänomenen im Kontext der allgemeinen historischen Entwicklung zugunsten einer falsch verstandenen Systematik geopfert würden, die der Frage nach der kulturgeschichtlichen Dimension des Memoriabegriffs nicht adäquat wäre.

Der hier verfolgte Ansatz, räumlich vermittelte Praktiken und Inszenierungen der Heiligenerinnerung auf Formen der kollektiven Identitätsstiftung zu beziehen, hat daher notwendigerweise einen fallstudienartigen Charakter, der eine struktur- und entwicklungsgeschichtliche Herangehensweise miteinander zu verbinden sucht. Konkret bedeutet dies, dass einzelne Felder kollektiver Identitätsstiftung nicht über den gesamten hier untersuchten Zeitraum von der Mitte des 3. bis zum Ende des 5. Jh. hinweg verfolgt werden, sondern nur für diejenigen Zeiträume, in denen sie in eine historisch bedeutsame Entwicklung eintreten und dadurch in der Regel auch quellenmäßig besser

86 Zu dieser Tendenz der aufklärerischen Geschichtsschreibung, Geschichte als einen universalen Prozess mit teleologischem Richtungssinn zu verstehen und damit das Erbe der christlichen Heilsgeschichte in säkularisierter Form anzutreten, vgl. die instruktiven Bemerkungen von Timpe 2001, 1-15. 
fassbar werden. Der folgende Abriss vermittelt in knapper Form eine Übersicht über die einzelnen Teilkapitel und verdeutlicht, in welcher Weise der Charakter der Quellenüberlieferung und der Kontext der historischen Entwicklung die Auswahl und Anordnung der einzelnen Themen bestimmt haben.

Als das früheste römische Zeugnis für räumlich vermittelte Formen kollektiver Identitätsstiftung durch Heiligenerinnerung kann die sogenannte „Triklia“ gelten - eine Kultstätte für die Apostel Petrus und Paulus an der via Appia aus der 2. Hälfte des 3. Jh. Der archäologische und epigraphische Befund der Anlage führt in die Welt kaiserzeitlicher Vereinsbildungen, die das Gedenken an ihre verstorbenen Mitglieder aufrecht erhielten: Die in der Kultstätte an der via Appia gefunden Graffiti eröffnen Einblicke in die identitätsstiftende Wirkung der Heiligenerinnerung für soziale Gruppen, die den collegia der hohen Kaiserzeit ähneln. Dabei liefern die Graffiti nur geringen Aufschluss hinsichtlich der sozialen Zusammensetzung der Gruppen, die sich zum Gedenken der Apostel in der Kultstätte an der via Appia zusammenfanden. Ihre eigentliche Bedeutung liegt darin, dass die in ihnen thematisierte Relation der Lebenden zu den Toten Spezifika der christlichen Memorialkultur hervortreten lässt, die grundlegende Unterschiede zum nicht-christlichen Totengedenken, wie es in den kaiserzeitlichen Vereinen gepflegt wurde, aufweist. Die Zeugnisse der Besucher der „Triklia“ sind daher nicht allein für das spätere 3. Jh. von Interesse, sondern offenbaren ein kulturelles Strukturphänomen „mittlerer Dauer“, 87 dessen geringe Entwicklungsgeschwindigkeit gleichsam den Grundton für den weiteren Gang der Untersuchung bildet (Kapitel II).

In eine Beziehung zur Stadt und zur Bürgeridentität gerieten die Orte der römischen Heiligentopographie erstmals unter Kaiser Konstantin (306-337), der durch die Errichtung von mehreren Basiliken in den Coemeterien des römischen Suburbiums den christlichen Toten- und Heiligenkult zu einem Teil der monumentalen Landschaft Roms machte. Da es sich beim Herren dieses Bauprogramms um den Kaiser handelte, der im räumlichen Kontext „seiner“ Stadt Rom ${ }^{88}$ agierte, werfen Konstantins monumentale Basilikalbauten zwangsläufig die Frage auf, ob und in welcher Weise der Kaiser die neue Religion in die urbs Roma integrierte. Eine Antwort auf diese Frage verlangt

87 In Anlehnung an Braudels Differenzierung von drei Ebenen unterschiedlicher historischer Entwicklungsgeschwindigkeit: politische Ereignisse (conjunctures) - soziale und wirtschaftliche Strukturen (structures) - geographisch-klimatische Rahmenbedingungen der „longue durée“ (vgl. Braudel 1990, Bd. 1, 20f.; bilanzierend zum Ansatz Daniel 2001, 223f.).

88 Zu dieser Relation zwischen dem Kaiser und der Stadt Rom vgl. Veyne 1990 (1976), 441-632; Flaig 1992, 38-93. Diese besondere Beziehung blieb auch nach der Distanzierung der Kaiser vom alten caput imperii während des 3. Jh. und der Begründung neuer Residenzen seit der tetrarchischen Zeit zunächst anschauungsleitend; erst um 400 trat Konstantinopel mit dem Anspruch auf, eine neue Haupt- und Kaiserstadt des Römischen Reichs zu sein. 
eine Ausweitung der Perspektive über den Heiligen- und Totenkult hinaus: Inwieweit Konstantin den christlichen Heiligen- und Totenkult zum Teil der städtischen Identität Roms machte oder ihn als eine kaiserliche Privatangelegenheit betrachtete, macht sich wesentlich daran fest, ob der Kaiser in seiner Kommunikation mit den unterschiedlichen Gruppen der Stadt auch voneinander getrennte Identitätskonstruktionen - der Kaiser und „seine Stadt“ Rom, der Kaiser und die christliche Gemeinde Roms - vermittelte. Die Monumentalisierung des christlichen Erinnerungsraums in den Coemeterien des römischen Suburbiums kann demnach nicht isoliert betrachtet, sondern muss in einem weiteren Kontext gesehen werden, der auch andere identitätsrelevante städtische Räume und Handlungsbereiche im spätantiken Rom mit einschließt. Aus diesem Grund gliedert sich das entsprechende Teilkapitel in zwei große Abschnitte. Der erste Teil untersucht die konstantinischen Bauten im Zentrum der Stadt und die auf die urbs Roma bezogene Kommunikation, die der Kaiser dort inszenierte. Der zweite Abschnitt verfolgt dieselbe Frage mit Blick auf die Coemeterialarchitektur, die Konstantin außerhalb der Stadt errichten ließ. Dabei wird zum einen deutlich werden, dass Konstantin zwar die unterschiedlichen Räume der Stadt nutzte, um mit verschiedenen Gruppen zu kommunizieren, damit jedoch keine voneinander getrennten Identitäten begründete: Die Kommunikation im Zentrum und in den Außenbezirken der Stadt betraf unterschiedliche Aspekte einer umfassenden kollektiven Identität, die durch die Relation des Kaisers zu seiner Stadt gegeben war. Zum anderen ist dieses Ergebnis auch ein Beitrag zum umfassenderen Komplex der mit der Hinwendung Konstantins zum Christentum verbundenen Forschungsprobleme: Die Monumentalisierungen, die Konstantin in der Stadt Rom und ihrem Suburbium betrieb, machen in exemplarischer Weise deutlich, wie sehr jede Beurteilung der Christianisierung Konstantins von zwei zentralen Faktoren ausgehen muss - den kulturellen Voraussetzungen, die Konstantins Verständnis von Christentum prägten, und der Frage nach den unterschiedlichen kommunikativen Kontexten, in denen seine Hinwendung zum Christentum öffentlichkeitswirksam wurde (Kapitel III).

Ab der Mitte des 4. Jh. beginnen Identitätskonflikte innerhalb der christlichen Gemeinde Roms - schismatische und häretische Spaltungen, die zwar auch schon in vorkonstantinischer Zeit stark ausgeprägt waren, jedoch in ihren topographischen Aspekten und in ihrer Relation zur Heiligenmemoria erst seit dem 4. Jh. fassbar werden - in das historische Blickfeld des Betrachters zu treten. Mehrere schismatische und häretische Konfliktsituationen des späteren 4. und frühen 5. Jh. verdeutlichen übereinstimmend, dass die konkurrierenden Anwärter auf die römische Cathedra ihre Legitimität vor allem dadurch zu untermauern suchten, dass sie als bischöfliche Leiter der Gemeinde, als Hirten ihrer Herde auftraten. Auch die Strategien der Raumbesetzung zielten dementsprechend darauf ab, sich als die wahre Bischofskirche zu 
präsentieren. Das kulturelle Leitbild vom Bischof inmitten seiner Herde verlangte nach symbolischer Präsenz innerhalb der Stadt, vorzugsweise in den Bischofskirchen, die im Unterschied zu den presbyteralen Titelkirchen die Identität der römischen Gemeinde in besonderer Weise verkörperten. Die suburbane Heiligentopographie war aufgrund ihrer räumlichen Randlage außerhalb der Stadtmauern hingegen wenig geeignet, das Bild von der Mitte der Gemeinde zu evozieren. Legitimierende Bezugnahmen auf individuelle heilige Patrone und Traditionsbezüge auf die apostolischen Gründer der Gemeinde spielten dementsprechend für die Legitimierung der römischen Bischofskandidaten keine erkennbare Rolle. Auch die umfassende Monumentalisierung der Heiligentopographie, die Bischof Damasus (366-384) vorantrieb, lief auf eine symbolische Eingliederung der Heiligen in seine Gemeinde, nicht auf eine Unterstellung des Bischofs unter den mächtigen Schutz heiliger Patrone hinaus. Damasus betrieb seine „Einbürgerung“ der römischen Märtyrer über eine politische und kulturelle Semantik, die sich an traditionellen Modellen städtischer Identitätsstiftung orientierte. Damit wurden die Heiligen zugleich zu Bezugspunkten von kollektiven Identitätsdiskursen, die nicht allein die römische Ortskirche betrafen. Vermittels der Heiligen machte Damasus das symbolische Kapital des caput mundi für die Vorrangstellung der römischen Kirche und ihres Leiters innerhalb der Reichskirche verfügbar - eine durch die damasianischen Epigramme auf den Heiligengräbern kommunizierte Botschaft, die aufgrund des seit dem späteren 4. Jh. stark anwachsenden Pilgerverkehrs auch ein außerrömisches Publikum erreichte. Mit seiner romanisierenden Übertragung der Semantik der urbs Roma in einen christlichen Kommunikationszusammenhang erwies sich Damasus gleichzeitig auch als der Wegbereiter einer christlichen Transformation städtischer Identität. Dass die Heiligen und die suburbane Märtyrertopographie in theodosianischer Zeit zu Symbolen der städtischen Gemeinde Roms wurden, blieb allerdings nicht Damasus selbst, sondern der auf ihn folgenden Generation vorbehalten (Kapitel IV).

Die Heiligen entwickelten sich seit dem späteren 4. Jh. nicht nur zu identitätsstiftenden Bezugspunkten der römischen ecclesia, der Universalkirche und der städtischen Gemeinde Roms. Hatten die Anfänge einer christlichen Sakraltopographie nach der „konstantinischen Wende“ auf den Baumaßnahmen Konstantins und der kaiserlichen Familie sowie des römischen Klerus beruht, gewannen im weiteren Verlauf des 4. Jh. die Initiativen der unter Konstantin reorganisierten Senatsaristokratie zunehmend an Bedeutung. Deren Einfluss machte sich vor allem bei der Errichtung von Kirchen bemerkbar: Zwischen dem letzten Drittel des 4. und dem ersten Drittel des 5. Jh. wurden zahlreiche römische Wohnhäuser von aristokratischen Stiftern in Kirchen umgewandelt und verdeutlichen das Ausmaß, mit dem die Senatsaristokratie in dieser Zeit den Ausbau der christlichen Sakraltopographie Roms vorantrieb. Angesichts 
der traditionellen Bedeutung der römischen domus als Ort der sozialen Repräsentation und politischen Kommunikation stellt sich die Frage, ob und in welcher Form die Heiligenverehrung in die öffentlichkeitsorientierte Architektur der domus und die soziale Struktur der Häuser integrierbar war: Welche Bedeutung hatte die Lokalisierung von Heiligenkulten und Reliquien in aristokratischen Haushalten, die zugleich die Zentren einer durch ausgeprägte Patronagebeziehungen strukturierten Gesellschaft bildeten und als Räume einer politischen Kommunikation fungierten, in denen die Hausbesitzer gegenüber Klienten und Standesgenossen Status und Sozialprestige in öffentlichkeitswirksamer Weise inszenierten? Insbesondere mit Blick auf die zeitgleiche Entwicklung in Konstantinopel ist ferner zu berücksichtigen, inwieweit Orte der Heiligenverehrung außerhalb des räumlichen Kontextes der domus der Kontrolle und Fürsorge durch vermögende und einflussreiche Angehörige der Oberschicht unterlagen und in welchem Maße die Heiligenverehrung Formen kollektiver Identitätsstiftung begünstigte, die für die gesellschaftlichpolitische Struktur der römischen Bürgerschaft konstitutiv waren (Kapitel V).

In demographischer und urbanistischer Hinsicht brachte das 5. Jh. für die Stadt Rom einschneidende Veränderungen mit sich. Einhergehend mit einem rapiden Rückgang der Einwohnerzahl machte das bis dahin geschlossene Stadtbild einer immer stärkeren Nuklearisierung Platz, in der sich die Textur des städtischen Raums durch die Auflassung von Wohnbereichen zu lockern begann. Diese siedlungstopographische und urbanistische Entwicklung wirkte sich auch auf die christliche Sakral- und Heiligentopographie aus: Rom wurde zum Schauplatz einer Stationsliturgie, in der die einzelnen Kirchen mit ihren Heiligenpatrozinien isolierte Bezugspunkte in einer neu entstehenden städtischen Landschaft bildeten. Parallel dazu erfuhr auch der Kirchenraum selbst eine vergleichbare Parzellierung: Die Zunahme an Messen und mit Reliquien ausgestatteter Altäre bewirkte eine Fragmentierung liturgischer Formen und kulttopographischer Elemente, die bis dahin als ein Sinnbild der Einheit der Gemeinde gegolten hatten. Sowohl die Nuklearisierung des städtischen als auch die Fragmentierung des liturgischen Raums, die während des 5. Jh. erst in Ansätzen fassbar werden, prägten sich im Laufe der nachfolgenden Entwicklung während des 6. Jh. weiter aus - eine Entwicklung, die es nahe legt, unter diesem Gesichtspunkt von einem langen 5. Jh. zu sprechen. Die genannten Tendenzen spiegelten sich auch in der Wahrnehmung Roms wider, dem literarischen „Rome imaginaire“, das die römischen Märtyrerlegenden, die sich im Verlauf des 5. Jh. zu einer aufblühenden Literaturgattung entwickelten, entwerfen und in dem eine spezifische Vorstellung vom städtischen Raum vermittelt wird. In den römischen gesta martyrum zerfällt die Stadt in eine Vielzahl mit der Erinnerung an das Leben einzelner Heiliger verbundener topographischer Punkte, die keinen städtischen Zusammenhang mehr bilden. In Form einer intensiv ausgeprägten literarischen Kommunikation, die 
sich in einer wechselseitigen Beeinflussung der einzelnen Legenden durch Wandermotive und stereotype Motivbildungen äußerte, formte sich damit ein Bild Roms, das von ähnlichen Nuklearisierungstendenzen geprägt war wie der städtische und der liturgische Raum. Für das Gros der hagiographischen Produkte ist dieses Phänomen nur auf der textimmanenten Ebene des Diskursiven und der literarischen Kommunikation fassbar; einige dieser Legenden ermöglichen jedoch eine präzise produktions- und rezeptionsästhetische Verortung im Schisma zwischen Laurentius und Symmachus (498-502/506), in dessen Kontext ein früheres Schisma zwischen Liberius und Felix II. hagiographisch überformt wurde. Ähnlich wie Laurentius und Symmachus bedienen sich auch die Akteure der Legenden topographischer Erinnerungsbezüge, um ihren Anspruch auf den römischen Bischofsthron zu legitimieren. Dieses Beispiel verdeutlicht die enge innere Verbindung zwischen Text und historischem Kontext und zeigt exemplarisch, dass die Imagination Roms in den gesta martyrum zwar das Produkt einer literarischen Kommunikation ist, in die jedoch Wahrnehmungen des städtischen Raums durch die Zeitgenossen Eingang gefunden haben (Kapitel VI).

Durch die Themen der einzelnen Teilkapitel lassen sich eine begründete Auswahl an kollektiven Identitäten, die mit Hinblick auf die identitätsstiftende Funktion der Heiligenmemoria diskutiert werden - die Gemeinschaft der Lebenden und Toten, die städtische Gemeinde der urbs Roma, die römische ecclesia in ihren lokalen und reichsweiten Bezügen, die soziale Institution der domus, die durch die römischen Heiligenlegenden konstituierte literarische Öffentlichkeit - und eine entwicklungsgeschichtliche Perspektive in überzeugender Weise miteinander verbinden. Wie bereits hervorgehoben, ist das Ergebnis nicht „die“ Geschichte der Heiligenmemoria im spätantiken Rom. Sie ermöglicht es aber durchaus, allgemeine Entwicklungstendenzen zu verdeutlichen, die in einer abschließenden Beurteilung auf strukturelle Eigentümlichkeiten in der christlichen Konzeptualisierung von Memoria hin untersucht werden - Eigentümlichkeiten, die die kulturspezifische Dimension der Erinnerung hervortreten lassen und einen wesentlichen Faktor für einen kulturellen Wandel im Übergang zum Frühmittelalter gebildet haben. Den Abschluss der Arbeit bildet daher ein über den engeren Rahmen des Heiligen- und Totengedenkens und über die einzelnen Fallstudien hinausführender Vergleich der unterschiedlichen Handlungsbeziehungen, die durch Memoria in der antiken Kultur und im Christentum begründet wurden: Ausgehend von der Beobachtung, dass das Erinnern als soziales Handeln Teil einer Gabentauschbeziehung ist, werden die strukturellen Unterschiede zwischen antiker und christlicher Konzeptualisierung des Gebens herausgestellt und die Konsequenzen verdeutlicht, die aus diesen unterschiedlichen Vorstellungen eines Gabentauschs für die Formierung kollektiver Identität durch Memoria erwuchsen. Die Auswirkungen dieses Wandels machten sich vor allem in der 
historischen Formation bemerkbar, für deren Existenz ein memorialer Gabentausch eine konstitutive Bedeutung hatte: der antiken Stadt (Kapitel VII).

Abschließend erscheint noch eine kurze Bemerkung zum Untersuchungszeitraum und der zeitlichen Eingrenzung der Thematik angebracht. Dass die Studie bereits mit der Mitte des 3. Jh. und damit noch vor der tetrarchischen Zeit einsetzt, die in den Handbüchern üblicherweise als eine epochale Zäsur zwischen der hohen Kaiserzeit und der Spätantike erscheint, trägt dem Umstand Rechnung, dass die gängige, an der politischen Geschichte orientierte Periodisierung für das Thema der Heiligenmemoria keine sinnvolle Grundlage bildet. Für die Geschichte der Heiligenverehrung sind vielmehr die ersten Ansätze zu einer kirchlichen Organisation der Heiligenkommemoration in der ersten Hälfte des 3. Jh. ${ }^{89}$ und vor allem die beiden Verfolgungen aus der Mitte des 3. Jh. - unter den Kaisern Decius (249-251) und Valerian (257/258) - von zentraler Bedeutung gewesen. Dass das Märtyrer- und Totengedenken in dieser Zeit eine identitätsstiftende Wirkung erlangte, wird unmittelbar daraus ersichtlich, dass die kaiserliche Gesetzgebung erstmals seit der valerianischen Verfolgung ein Versammlungsverbot in den christlichen Kommemorationsstätten - den sogenannten Coemeterien - auszusprechen begann. ${ }^{90}$ Dieses intensivierte Toten- und Heiligengedenken, auf das die kaiserlichen Verbote reagierten, ist nicht allein der Tatsache zuzuschreiben, dass durch die Verfolgungen der 250er Jahre, die erstmals in der Geschichte der alten Kirche reichsweit durchgeführt und zentral organisiert wurden, ${ }^{91}$ vermutlich ein Anstieg der bis dahin wohl eher bescheidenen Zahl an Märtyrern und Confesso-

$89 \quad$ Baumeister 1988, 116-122.

90 Vgl. das Edikt Valerians mit dem Verbot, Versammlungen abzuhalten und die ,,sogenannten

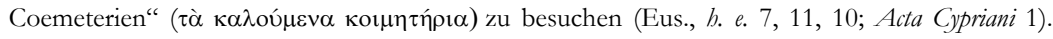
Das Restitutionsedikt des Gallienus sah vor, dass die Bischöfe die mit den Coemeterien verbundenen Areale wieder in Besitz nehmen durften (Eus., b. e. 7, 13). Maximinus Daia verhinderte im Jahr 311 erneut, dass die Christen sich in ihren Coemeterien versammelten (Eus., h. e. 9, 2). Was der Begriff коцити́рıо/coemeterium genau bedeutet, ist umstritten. Ein Teil der Forschung

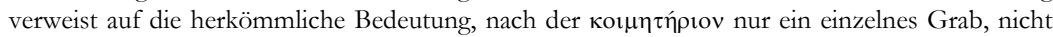
aber eine kollektive Grablege bezeichnet, und deutet die kaiserlichen Edikte als Versammlungsverbote an den Heiligengräbern (Rebillard 1993, bes. 981f.; ders. 2003, 115); dagegen steht die traditionell vertretene Auffassung, wonach die Coemeterien Grabareale darstellen, die sich in Gemeinde- oder - bis zum Restitutionsedikt des Gallienus - auch in Privatbesitz befanden und zur Bestattung christlicher Gemeindemitglieder bestimmt waren (so Brandenburg 1994, 209f., Fiocchi Nicolai 2003, 380f.). Insbesondere die Situation in Rom, wo mit dem coemeterium Callisti

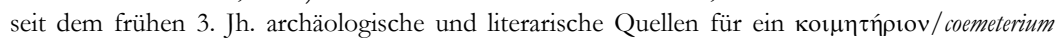
vorliegen, die auf eine gemeindeeigene kollektive Grablege hinweisen, legt nahe, dass die Terminologie nicht auf Märtyrergräber zu beziehen ist (vgl. auch u., S. 39). Auch wenn die kaiserlichen Versammlungsverbote in den Coemeterien daher nicht ausschließlich auf Heiligengräber abzielen, signalisieren sie in jedem Fall eine gestiegene Bedeutung des Totenkults und des Märtyrergedenkens.

91 Auf die unterschiedlichen Motive der decischen und valerianischen Verfolgungen ist hier nicht näher einzugehen; eine konzise Analyse bietet Molthagen 21975, 61-100. 
ren zu verzeichnen war. ${ }^{92}$ Vielmehr entwickelte sich, bedingt durch den Umstand, dass die während der Verfolgung abgefallenen Gemeindemitglieder, die sogenannten lapsi, bei den in den Gefängnissen auf ihre Verurteilung wartenden Märtyrern und Bekennern zum Teil auf eine vergebungsbereitere Haltung stießen als bei der Amtskirche, noch zu Lebzeiten der Heiligen eine intensive Kommunikation zwischen ihnen und ihren Besuchern, die sich in Form des Kultes für den verstorbenen Märtyrer über die Grenze des Todes hinweg fortsetzte und Bestand hatte. ${ }^{93}$ Dass Lebende und Tote durch einen reziproken Handlungszusammenhang miteinander verbunden blieben, stellt ein Spezifikum der christlichen Memorialkultur dar, das in den literarischen und archäologischen Quellen des 3. Jh. erstmals deutlich zutage tritt und für die weitere Entwicklung des Heiligenkultes von fundamentaler Bedeutung gewesen ist. Obwohl ihre Anfänge bis in das frühe 2. Jh. zurückreichen, ${ }^{94}$ bildet das 3. Jh. somit in mehrfacher Hinsicht eine formative Entwicklungsphase in der Geschichte der Heiligenmemoria - sowohl mit Blick auf die Dimensionen, die sie innerhalb der christlichen Erinnerungskultur erlangte, als auch vor allem hinsichtlich der Art und Weise, in der die Memorialbeziehungen zwischen den Heiligen und ihren Verehrern strukturiert waren und Identität in der Erinnerung an den Heiligen begründeten.

Auch der Abschluss der Studie um das Jahr 500 orientiert sich nicht an den gängigen Epochengrenzen. Üblicherweise wird die Geschichte des spätantiken Rom erst etwa ein Jahrhundert später als beendet angesehen, mit der Person Gregors I. (590-604), unter dem die Institutionen des römischen Senats und der Stadtpräfektur letztmalig erwähnt werden und Rom, das alte caput imperii, sich von einem imperialen zu einem kirchlichen Zentrum und Ordnungsfaktor für die germanischen Reiche, die auf dem Boden des

92 Die römische Ordnungsmacht war bis zur Mitte des 3. Jh. an der Vermeidung von Konflikten interessiert und bemüht, Martyrien zu verhindern: Bereits die durch Trajan autorisierte formale Festlegung auf das nomen Christianum als Verurteilungsgrundlage - unter Absehung einer Verfolgung begangener Straftaten - ist als ein Versuch zu bewerten, die ordnungspolitischen Verwerfungen durch Christenprozesse möglichst gering zu halten (so auch Bringmann 1978). Ob dies bedeutet, dass Martyrien bis zur Mitte des 3. Jh. eine vergleichsweise seltene Erscheinung waren und erst die staatlichen Verfolgungen zu einem signifikanten Anstieg führten, ist in der Forschung umstritten (mit einem Anstieg rechnen z. B. Frend 1965, 413; Hopkins 1998, 195-198; zurückhaltender urteilt Lane Fox 1986, 450-462; Lepelley 2003, 256f. wiederum veranschlagt bereits für die Zeit vor 250 sehr hohe Zahlen an Märtyrern). Jenseits dieser rein numerischen Frage ist jedoch sicher, dass durch die Edikte des Decius und Valerians die Fragen von Martyrium und Verfolgung zu einem alle Gemeinden betreffenden Thema wurden - was wiederum zur Folge hatte, dass sich Märtyrer und Martyrien zu einem wesentlichen Aspekt des Gemeindelebens entwickelten (vgl. das folgende).

93 S. dazu ausführlich u., S. 68-77.

94 Der früheste Beleg für ein regelmäßig stattfindendes Heiligengedenken ist M. Polyc. 18, 3 (vermutlich um $167 \mathrm{zu}$ datieren). 
imperium Romanum entstanden waren, zu wandeln begann. ${ }^{95}$ Mit Blick auf die Entwicklung der römischen Heiligenverehrung hatte Gregors Pontifikat jedoch keinen Zäsurcharakter. Einer der wichtigsten Anstöße zur Umgestaltung der Heiligentopographie im römischen Suburbium - die Errichtung von ad corpus-Basiliken über den Gräbern der Heiligen, die an die Stelle der älteren Umgangsbasiliken traten, - ging vielmehr bereits von Gregors unmittelbarem Vorgänger, Pelagius II. (579-590), aus und wurde von Gregors Nachfolger Honorius I. (625-638) fortgeführt. ${ }^{96}$ Honorius errichtete zudem noch zahlreiche weitere Sakralbauten über den Heiligengräbern im römischen Suburbium ${ }^{97}$ und entfaltete damit Initiativen zur Förderung des Heiligenkults, die auch Spuren in der römischen Bischofsliturgie hinterließen ${ }^{98}$ und den Anlass zur Entstehung von Pilgerführern gaben, die den Besuchern Roms die Märty-

$95 \quad$ Zur letzten Bezeugung des römischen Senats und der Stadtpräfektur unter Gregor I. und zum Verschwinden der Institutionen vgl. Arnaldi 1982, bes. 6-12, 34-36. Für Gregors Beitrag zur Loslösung Roms aus der reichskirchlichen Struktur des (ost-)römischen Reiches und zur kirchlichen Transformation des römischen Universalismus, der nun wesentlich auf der Petrusfrömmigkeit und auf den missionarischen und kirchenrechtlichen Beziehungen beruhte, die der Bischof von Rom zu den germanischen Königreichen des Westens unterhielt, vgl. Ullmann 1955, 36-38. Caspar 1930/1933, Bd. 2, 471-514 urteilt in dieser Hinsicht zurückhaltender und bemisst die epochale Bedeutung von Gregors Pontifikat vor allem an der asketischen Persönlichkeit des römischen Bischofs, die das Kennzeichen einer kulturell-geistigen Zeitenwende gewesen sei.

96 Pelagius II. errichtete eine ad corpus-Basilika für den Hl. Laurentius an der via Tiburtina, Honorius einen vergleichbaren Bau für die Hl. Agnes an der via Nomentana (vgl. zuletzt Brandenburg 2004, 236-248 mit der jüngsten Literatur). Beide Bauten ersetzten ältere Umgangsbasiliken, die keine unmittelbare Verbindung mit den Heiligengräbern aufwiesen (vgl. u., S. 163f.). Die diesen beiden Bauten zugrunde liegende Konzeption, eine unmittelbare Verbindung zwischen dem Altar und dem Heiligengrab herzustellen, war auch für die Umbaumaßnahmen in der vatikanischen Petrusbasilika und in S. Paolo f.l.m. maßgeblich, in denen die Altarbereiche erhöht und mit Ringbzw. Hallenkrypta versehen wurden, die das unter den jeweiligen Altären liegende Grab der Heiligen zugänglich machte (vgl. de Blaauw 1995, 560f.). Obwohl diese Umbauten in den beiden apostolischen Basiliken unter Gregor I. durchgeführt wurden, gilt es zumindest im Fall von S. Pietro als wahrscheinlich, dass die Pläne bereits auf Pelagius II. zurückgehen (Apollonj Ghetti u. a. 1951, Bd. 1, 176, 193; de Blaauw 1994, Bd. 2, 533f.).

97 Der Liber Pontificalis beschließt eine Auflistung der wichtigsten Projekte des Honorius mit der Bemerkung, dass es zu weit führen würde, alle seine Baumaßnahmen im einzelnen aufzuzählen (vgl. LP I, 324: multa alia fecit quas enumerare longum est). Von der intensiven Bautätigkeit des Honorius zeugen nicht nur die literarischen Nachrichten des Liber Pontificalis, sondern auch die frühmittelalterlichen Inschriftensammlungen, die sogenannten Syllogen, die zahlreiche Bau- und Dedikationsinschriften aus römischen Kirchen überliefert haben und in die eine außergewöhnlich hohe Zahl von Inschriften des Honorius aufgenommen wurden, vgl. de Rossi, Inscr. II, 1, XLII-XLV.

98 Der Hauptüberlieferungsstrang gregorianischen Sakramentars, der durch das Hadrianum und das Paduanum repräsentiert wird, geht auf eine Redaktion dieses Sakramentars unter Honorius zurück (vgl. Deshusses ${ }^{2} 1992$, Bd. 1, 50-60, bes. 50 u. 52). Dabei fanden zahlreiche Heiligenfeste von Kirchen, die Honorius gegründet hatte, Eingang in die Bischofsliturgie; dies wird sowohl aus der gregorianischen Sakramentartradition ersichtlich als auch aus den Perikopenordnungen mehrerer Evangeliare, die in der Zeit zwischen Honorius I. und Leo II. (682-683) verfasst wurden (vgl. die entsprechenden Bemerkungen von Baumstark, in: Mohlberg/Baumstark 1927, *90-96; ebd., *57f.). 
rergräber und Kirchen Roms in Rundgängen durch die Stadt und ihr Suburbium erschlossen. ${ }^{99}$ Auch sonst vollzogen sich entscheidende Entwicklungen auf dem Gebiet der römischen Heiligenverehrung und -topographie in der Zeit vor und nach Gregor I. Seit der Mitte des 7. Jh. und verstärkt seit Paul I. (757-767) wurden die sterblichen Überreste der Heiligen aus den Katakomben in die städtischen Kirchen überführt; ${ }^{100}$ schon deutlich früher, seit dem Beginn des 6. Jh., hatten jedoch erste Tendenzen eingesetzt, die liturgische Einheit des Kirchenraums durch die Anlage von Heiligenkapellen aufzubrechen. ${ }^{101}$ Die römische Hagiographie, die sich während des 5. Jh. zu entfalten begann, wurde seit dem 8. Jh. in Martyrologe und Legendare integriert, die nicht in Rom selbst, sondern in der fränkischen Welt ins Leben gerufen wurden und den umfangreichen Bestand an römischen Heiligenlegenden in neue Literaturgattungen überführten, über deren Vermittlung sie sich von da an im früh- und hochmittelalterlichen Europa rasch verbreiteten. ${ }^{102}$ In Anbetracht dieser weit gespannten Entwicklungslinien, die durch Gregor I. nicht wesentlich beeinflusst wurden, erschien es sinnvoll, den Schlusspunkt dieser Studie nicht erst mit der Wende vom 5. zum 6. Jh., sondern bereits mit Symmachus (498-514) zu setzen. Maßgeblich für diese Periodisierung ist dabei nicht so sehr die Auffassung, dass mit Symmachus eine Entwicklung zum Abschluss käme, als vielmehr die Erkenntnis, dass während seines Pontifikats erstmals Phänomene deutlich und präzise fassbar werden, die einen grundlegenden Strukturwandel erkennen lassen und über die Wende vom 4. zum 5. Jh. hinaus auf die frühmittelalterliche Entwicklung verweisen. Die im Rombild der Heiligenlegenden und in der bischöflichen Stationsliturgie sich etwa seit der Mitte des 5. Jh. abzeichnende Nuklearisierung des städtischen Raums durch eine polyzentrische Heiligentopographie findet im Schisma zwischen Symmachus und Laurentius, in dem die legitimatorische Funktion dieser Zentren auf den unterschiedlichsten Ebenen (Besetzung des städtischen Raums, Hagiographie, Gestaltung von Kirchenräumen) ausgespielt wird, einen exemplarischen Ausdruck: In der Zeit um 500 war die Schwelle zu einer neuen Zeit bereits überschritten.

\footnotetext{
99 Es sind mehrere solcher frühmittelalterlichen Itinerare erhalten, von denen die notitia ecclesiarum urbis Romae und das weniger ausführliche de locis sanctis martyrum von der Forschung traditionell in die Zeit von Honorius I. (notitia) oder kurz danach (de locis) datiert werden, vgl. de Rossi 18641877, Bd. 1., 144f., der die beiden erhaltenen Itinerare auf eine verlorene Schrift aus der Zeit von Honorius I. zurückführt (ebd. 152f.); vgl. ferner Valentini/Zucchetti (1942), in: notitia ecclesiarum, 69f.; ebd. (de locis), 102f.; Bauer 1997 (a), 217f.

100 Ihren Höhepunkt erreichte diese Entwicklung erst in der ersten Hälfte des 9. Jh., vgl. den Überblick bei Osborne 1985, 286-295.

101 Bauer 1999 und u., S. 415-424.

102 Philippart 1977, 27-36; Pilsworth 2000; Diesenberger 2006.
} 